\title{
Computational nonlinear stochastic homogenization using a non-concurrent multiscale approach for hyperelastic heterogeneous microstructures analysis
}

\author{
A. Clément, C. Soize *, J. Yvonnet \\ Université Paris-Est, Laboratoire Modélisation et Simulation Multi Echelle, MSME UMR 8208 CNRS, 5 Bd Descartes, \\ 77454 Marne-la-Vallée, France
}

\begin{abstract}
SUMMARY
This paper is devoted to the computational nonlinear stochastic homogenization of a hyperelastic heterogeneous microstructure using a non-concurrent multiscale approach. The geometry of the microstructure is random. The non-concurrent multiscale approach for micro-macro nonlinear mechanics is extended to the stochastic case. Since the non-concurrent multiscale approach is based on the use of a tensorial decomposition, which is then submitted to the curse of dimensionality, we perform an analysis with respect to the stochastic dimension. The technique uses a database describing the strain energy density function (potential) in both the macroscopic Cauchy Green strain space and the geometrical random parameters domain. Each value of the potential is numerically computed by means of the finit element method on an elementary cell whose geometry is given by the random parameters and the corresponding macroscopic strains being prescribed as boundary conditions. An interpolation scheme is finall introduced to obtain a continuous explicit form of the potential, which, by derivation, allows to evaluate the macroscopic stress and elastic tangent tensors during the macroscopic structural computations. Two numerical examples are presented
\end{abstract}

KEY WORDS: computational mechanics; stochastic homogenization; nonlinear heterogeneous materials; random microstructures

\section{INTRODUCTION}

When a structure is made of a highly heterogeneous material at the microscopic level, classical numerical methods used for structural analysis lead to prohibitive memory and computational costs and can hardly be used for standard engineering studies. Homogenization methods represent an effective alternative for determining the macroscopic behavior of such materials. However, the case of nonlinear heterogeneous materials leads us to a set of additional difficultie regarding the linear case: for instance, the general form of the constitutive law is not known and the principle of superposition does not hold which prevents using any analytical homogenization scheme introduced for the case of small elastic strains. To overcome these difficulties two classes of methods have been developed and used during the last two decades. The firs one consists in extending classical analytical homogenization approaches by derivation of estimate and exact solutions of some classes of hyperelastic composites $[1,2,3]$ or by introducing a second-order homogenization method to determine the effective constitutive laws of nonlinear composites (see $[4,5]$, for instance). The

*Correspondence to: Christian Soize, Université Paris-Est, 5 Bd Descartes, 77454 Marne-la-Vallée Cedex 2, France. 
second family of methods consists in using numerical multiscale simulations which can be an effective alternative approach when one seeks, for instance, to deal with complex microstructure. Then, concurrent multiscale methods were developed and aim at solving simultaneously the nonlinear problems at both scales (see for instance $[6,7,8,9,10,11,12,13])$. The macroscopic problem provides the strain states in all integration points and gives the boundary conditions used to solve each microscopic problem. This kind of techniques allows taking into account different types of nonlinearities but may leads to significan computational costs. Alternatively, the nonconcurrent multiscale methods aim at decoupling the numerical simulations at both scales. They consist in identifying parameters characterizing the macroscopic constitutive law from experimental or numerical test [14] or using a database formed of microscopic calculations to build a numerical constitutive law without any assumption on the constitutive equations $[15,16,17,18,19]$. In this work, we focus on the methodology proposed in $[17,18,19]$.

On the other hand, it may also be necessary to take into account the different sources of uncertainty at the microscopic level if one seeks to obtain a reliable model of the macroscopic behavior of the material. Thus, the general area of stochastic multiscale modeling of complex random heterogeneous microstructures has recently attracted a continuously growing attention in the scientifi community. A simple way to solve this kind of problem consists in coupling numerical simulations of random microstructures (based on experimental identificatio or some geometrical characteristics such that the volume fraction or others parameters such that the spatial and size distributions [20,21]) with classical finit element computations. Then, one can perform a statistical study of average quantities of interest characterizing the macroscopic behavior of the material (e.g. stress random fields) Recently, more sophisticated methods based on the Maximum Entropy principle have been developed in the case of linear elasticity. The so-called nonparametric approach for uncertainties aims at building prior probability distributions of effective stress tensors with no explicit representation of the random parameters characterizing the microstructure [22, 23, 24, 25]. Parametric approaches have also been used to characterize the macroscopic behavior. In [26, 27, 28], the authors have used spectral stochastic methods (e.g. polynomial chaos approximation) to solve the stochastic boundary value problem involved by the random microstructure. Monte-Carlo simulations may naturally be employed to perform a statistical study on the effective properties of the material (see for instance [29] and [30] where the Monte-Carlo approach is coupled with a $\mathrm{X}$-FEM methodology avoiding the remeshing difficulties)

The fundamental objective of the paper is to test a novel non-concurrent approach for solving a nonlinear stochastic homogenization problem. In this context, to the knowledge of the authors, the present paper is the first one devoted to this challenging issue. A choice concerning the mechanical nonlinearities of the microstructure must be made. There are two main types of mechanical nonlinearities: large deformations and displacements with linear constitutive equations (type I) and, small or large deformations with nonlinear constitutive equations (type II). As it the firs research in this fiel from the authors, we decide to adopt nonlinearities of type I which facilitate the definitio of the potential which is a crucial point of the proposed approach. Further works will be devoted to the extension of the method for different kinds of nonlinearities such as plasticity. On the other hand, the proposed method should, in the future, be applied to a practical example in the fiel of biomechanics such as the study of living tissues which follow a hyperelastic constitutive behavior. We here focus on fibe reinforced polymer composites, involving a condition of impenetrability between the inclusions, and on the geometrical uncertainties on the microstructure. The firs step of the method lies on the construction of a probabilistic model of the random microstructure. This issue has already been assessed by different authors in various ways. In [31, 32], the formalism of random sets is used to generate random heterogeneous microstructures while in [20, 33, 34] the same objective is done based on a Poisson random fiel model. We also propose to build a random generator of the microstructure which depends on a high number of random variables. We then use a classical statistical reduction through a Karhunen-Loeve expansion [35] which allows controlling the probabilistic model with a few number of reduced random variables. Numerous algorithms given in the literature may replaced the one develop in this work. However, the algorithm proposed in Section 3 allows introducing and controlling the correlation structure given to the 
geometrical parameters which makes effective the statistical reduction. Then, based on an particular nonlinear homogenization scheme, we propose to extend the method of the Numerical Explicit Potentials (NEXP method) $[17,18,19]$ to the stochastic framework. In the deterministic case, this approach is based on the numerical determination of the macroscopic strain energy density function (potential) according to the macroscopic strain state. Through a separated variables representation and an appropriate interpolation scheme, this approach allows obtaining an explicit form of the potential, which, by derivation, yields the stress and elastic tangent tensors which fully characterize the macroscopic constitutive law. In the stochastic framework, the aim is the same but the potential is not only related to the macroscopic strain state but also to the reduced random variables describing the random geometry of the microstructure. Then, the strain energy density function is depending on a set of parameters. This so-called parameters space is discretized into a sufficien number of points corresponding both to a particular macroscopic strain state and a particular geometry of the microstructure which involve a nonlinear boundary value problem numerically solved by a classical finit element method. In order to obtain an explicit form of the potential available for any points belonging to the parameters space, a separated variables representation associated to an appropriate interpolation scheme is adapted from $[18,19]$. Thus, the macroscopic structural computations can be easily and efficientl performed using the explicit form of the potential to determine the random macroscopic constitutive law.

Moreover, the probabilistic characterization of such a complex microstructure may require a high number of random variables. Thus, the question of the high dimensionality is also pointed out. We will then focus on the extension of this kind of numerical deterministic solvers to the stochastic framework when one seeks to use a high number of random variables to characterize the probabilistic model.

The paper is organized as follows. Section 2 deals with the homogenization of nonlinear heterogeneous materials at finit strains in a deterministic framework where both, microscale and macroscale problems are detailed. Section 3 presents the probabilistic model leading to a stochastic representation of the microstructure based on a parametric framework. We then detail, in Section 4, the proposed approach, called the method of Stochastic Numerical Explicit Potentials, which allows determining the constitutive law at the upper scale according to the random parameters characterizing the geometry of the microstructure. Numerical examples are finall provided in Section 5 in order to point the advantages and drawbacks of the proposed method. Some concluding remarks are finall drawn in the last section.

\section{DETERMINISTIC HOMOGENIZATION OF NONLINEAR HETEROGENEOUS MATERIALS}

\subsection{Nonlinear homogenization scheme}

We consider a microstructure, schematically represented on figur 1 , which occupies a domain $\Omega \subset \mathbb{R}^{d}$ where $d$ denotes the spatial dimension $(d \in\{1,2,3\})$. The boundary of the domain $\Omega$ is denoted by $\partial \Omega$ and the position of the material points are identifie by the vector $\boldsymbol{X}$ in the reference configuratio and by vector $\boldsymbol{x}$ in the deformed configuration These vectors are related through:

$$
\boldsymbol{x}=\boldsymbol{X}+\boldsymbol{u},
$$

where $\boldsymbol{u}$ is the displacement vector of a material point. We introduce the deformation gradient tensor $\boldsymbol{F}$ at point $\boldsymbol{X}$ define by

$$
\boldsymbol{F}=\frac{\partial \boldsymbol{x}}{\partial \boldsymbol{X}}=\mathbf{1}+\nabla_{X}(\boldsymbol{u})
$$

where 1 is the second-order identity tensor and $\nabla_{X}(\cdot)$ is the gradient operator according to the reference configuration The microstructure is associated with a multiphased heterogeneous material composed of $N_{p}$ hyperelastic phases definin the $N_{p}$ domains $\Omega^{(r)}$ with $r \in\left\{1, \ldots, N_{p}\right\}$ and such that $\Omega=\bigcup_{r=1}^{N_{p}} \Omega^{(r)}$. The constitutive equation of each phase can then be characterized by strain energy density functions (i.e. elastic potentials) $\psi^{(r)}$ according to the right-hand Cauchy-Green 


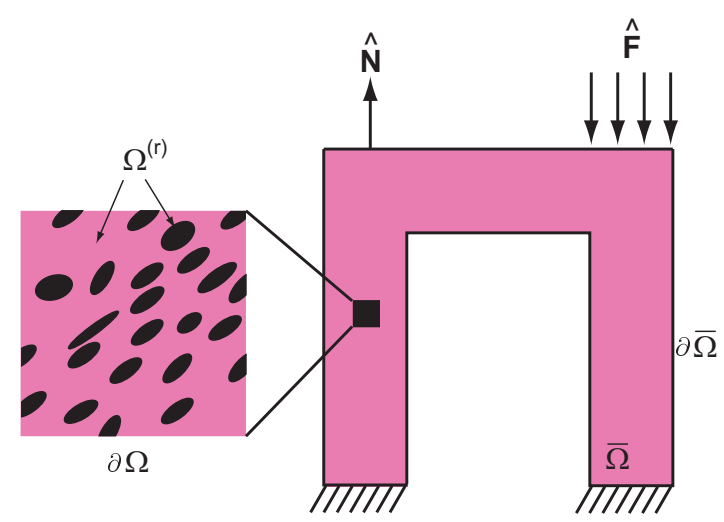

û

Figure 1. Model problem: microscopic and macroscopic structures

strain tensor $\boldsymbol{C}=\boldsymbol{F}^{T} \boldsymbol{F}$ such that the local strain energy density function $\psi$ of $\Omega$ may be written as

$$
\psi(\boldsymbol{X}, \boldsymbol{C})=\sum_{r=1}^{N_{p}} \mathbb{I}^{(r)}(\boldsymbol{X}) \psi^{(r)}(\boldsymbol{C}),
$$

where $\mathbb{I}^{(r)}$ is the characteristic function of domain $\Omega^{(r)}$ which is equal to 1 if $\boldsymbol{X} \in \Omega^{(r)}$ and 0 otherwise. The local constitutive law is given, in the Lagrangian description, by

$$
\boldsymbol{S}=2 \frac{\partial \psi}{\partial \boldsymbol{C}}(\boldsymbol{X}, \boldsymbol{C})
$$

where $S$ denotes the second Piola-Kirchhoff stress tensor. Several models are available for the hyperelastic constitutive law: we only here consider a compressible Neo-Hookean model described by the following potential

$$
\psi(\boldsymbol{C})=\frac{1}{2} \lambda\{\log (J)\}^{2}-\mu \log (J)+\frac{1}{2} \mu(\operatorname{tr}(\boldsymbol{C})-3),
$$

where $J=\operatorname{det}(\boldsymbol{F})$ is the volumetric change and where $\lambda=\frac{E \nu}{(1+\nu)(1-2 \nu)}$ and $\mu=\frac{E}{2(1+\nu)}$ with $E$ and $\nu$ respectively the Young modulus and the Poisson ratio. We also introduce the fourth-order tangent elastic tensor $\boldsymbol{L}$ define by

$$
\boldsymbol{L}=4 \frac{\partial^{2} \psi(\boldsymbol{X}, \boldsymbol{C})}{\partial \boldsymbol{C}^{2}} .
$$

We assume that the elementary cell is associated with a structure at a larger scale which can be a mesoscopic or a macroscopic scale depending on the separation between the considered scales. This issue will be discussed in Section 3. Thus, we defin the kinematic quantities as follows:

$$
\begin{aligned}
& \overline{\boldsymbol{F}}=\langle\boldsymbol{F}\rangle, \\
& \overline{\boldsymbol{C}}=\overline{\boldsymbol{F}}^{T} \overline{\boldsymbol{F}} \neq\langle\boldsymbol{C}\rangle,
\end{aligned}
$$

where symbol $<\cdot>$ denotes the spatial averaging over $\Omega$. The upper scale stress tensors are define according to

$$
\begin{aligned}
& \overline{\boldsymbol{P}}=\langle\boldsymbol{P}\rangle, \\
& \overline{\boldsymbol{S}}=\overline{\boldsymbol{F}}^{-1} \overline{\boldsymbol{P}} \neq\langle\boldsymbol{S}\rangle,
\end{aligned}
$$

where $\overline{\boldsymbol{P}}$ denotes the firs Piola-Kirchhoff macroscopic stress tensor. Using equations (7) to (10), it can be shown that

$$
\overline{\boldsymbol{S}}=2 \frac{\partial \bar{\psi}(\overline{\boldsymbol{C}})}{\partial \overline{\boldsymbol{C}}}
$$


where the effective strain energy density function $\bar{\psi}$ is define by

$$
\bar{\psi}(\overline{\boldsymbol{C}})=\inf _{\boldsymbol{C} \in \mathcal{K}(\overline{\boldsymbol{C}})}\langle\psi(\boldsymbol{X}, \boldsymbol{C})\rangle .
$$

In equation (12), the set $\mathcal{K}(\overline{\boldsymbol{C}})$ gathers all kinematically admissible strain tensors according to $\overline{\boldsymbol{C}}$. Thus, for a given strain state $\overline{\boldsymbol{C}}$, the corresponding value of $\psi(\overline{\boldsymbol{C}})$ is determined by taking the spatial average of local strain energy density functions $\psi(\boldsymbol{X}, \boldsymbol{C})$, where $\boldsymbol{C}(\boldsymbol{X})$ is the strain fiel at equilibrium. In a same manner, we can show that the tangent elastic tensor $\overline{\boldsymbol{L}}$ can be expressed according to $\bar{\psi}$ such that

$$
\overline{\boldsymbol{L}}=4 \frac{\partial^{2} \bar{\psi}(\overline{\boldsymbol{C}})}{\partial \overline{\boldsymbol{C}}^{2}} .
$$

Proofs yielding equations (11) and (13) which fully allow characterizing the upper scale constitutive law can be found in [19].

\subsection{Involved mechanical problems at different scales}

At the microscopic scale the stress must verify the local equilibrium condition:

$$
\nabla_{X} \cdot(\boldsymbol{F} \boldsymbol{S})=\mathbf{0} \quad \text { on } \Omega
$$

where $\nabla_{X} \cdot(\cdot)$ is the gradient operator according to the reference configuration Boundary conditions must be associated with equation (14) to obtain a unique solution. Several choices are possible: kinematically uniform, statically uniform or periodic boundary conditions. However, since the microstructure is characterized by random geometrical parameters, the implementation of periodic boundary conditions involves numerical difficultie since the finit element mesh is not regular on the boundary. This issue can be encountered using an X-FEM solver with adapted enrichment strategies such as the one proposed in [36] allowing multiple level-sets (i.e interfaces) cutting the same finit element. We therefore use linear boundary conditions expressed in case of finit strains:

$$
\boldsymbol{u}(\boldsymbol{X})=(\overline{\boldsymbol{F}}-\mathbf{1}) \boldsymbol{X} \quad \text { on } \quad \partial \Omega .
$$

At the upper scale, the equilibrium equation must be verified

$$
\nabla_{X} \cdot(\overline{\boldsymbol{F S}})=\mathbf{0} \text { on } \bar{\Omega},
$$

with the following boundary conditions

$$
\overline{\boldsymbol{u}}(\overline{\boldsymbol{X}})=\widehat{\boldsymbol{u}}(\boldsymbol{X}) \quad \text { on } \quad \partial \bar{\Omega}_{u}, \quad \overline{\boldsymbol{P}} \widehat{\boldsymbol{N}}=\widehat{\boldsymbol{F}} \quad \text { on } \quad \partial \bar{\Omega}_{F},
$$

where $\bar{\Omega}, \overline{\boldsymbol{u}}, \overline{\boldsymbol{X}}, \partial \bar{\Omega}_{u}, \partial \bar{\Omega}_{F}, \widehat{\boldsymbol{u}}(\boldsymbol{X}), \widehat{\boldsymbol{F}}$ and $\widehat{\boldsymbol{N}}$ are, respectively, the macroscopic domain, the macroscopic displacements, the macroscopic coordinates in the reference configuration the boundary of macroscopic Dirichlet conditions, the boundary of macroscopic Neumann boundary conditions, the applied displacements, external force and the outward unit normal vector to the boundary $\partial \bar{\Omega}$ of $\bar{\Omega}$. Both nonlinear problems define by equations (14) and (16) are classically linearized and solved with the Finite Element Method.

\section{PROBABILISTIC MODELING}

This section deals with the construction of the probabilistic model of the microstructure characterizing a fibe reinforced polymer composite. We firs introduce the chosen parametrization of the geometry. We then detail the probabilistic model consisting in a random generator combined with a classical statistical reduction through a principal component analysis. 


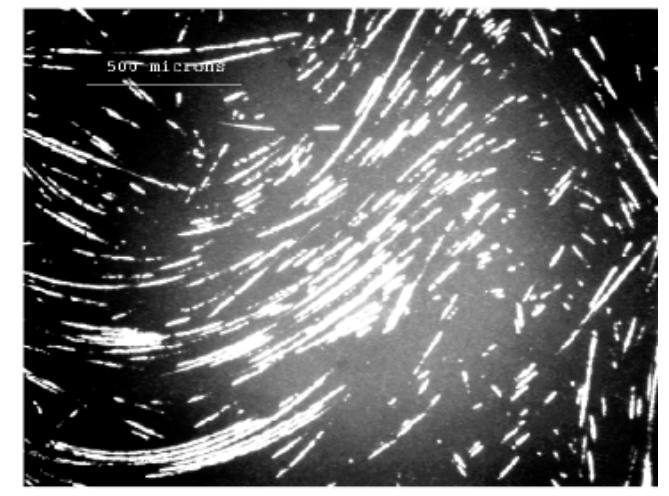

(a) 2D longitudinal section view.

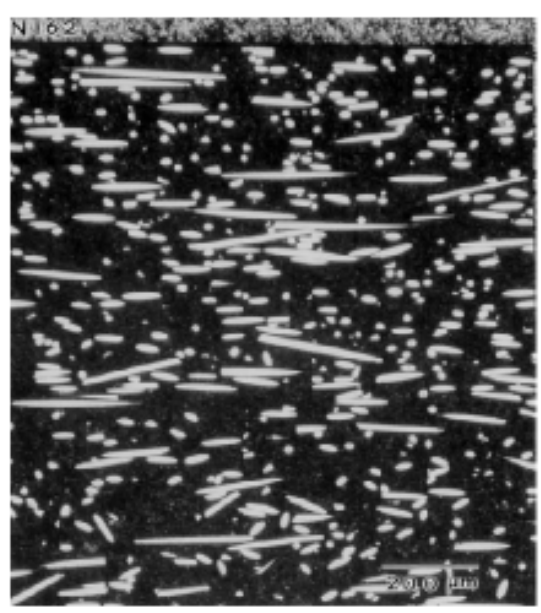

(b) $2 \mathrm{D}$ transversal section view.

Figure 2. 2D longitudinal and transversal section views of fibe reinforced polymer composites.

\subsection{Geometrical description of the microstructure}

This work is focussed on heterogeneous materials for which the geometrical parameters characterizing the microstructure are random. In particular, we are interested in composites made of polymer matrix and reinforced by non penetrating long fibers Manufacturing process of these kind of material involves uncertainties, related to the position of the fibers mainly due to the "fountain effect" of the injector. Moreover, since the 2D-cross section views, used for the microstructure mechanical modeling and for the mechanical numerical analysis, are coming from experimental data, they may involve error on the orientation of the fibers It thus leads us to another source of uncertainties. Figure 2 illustrates typical section views of this kind of material and clearly shows a correlation between the position and shape of the different fiber which characterize the probabilistic model. The geometrical modeling of the fiber is depicted on figur 3. The microstructural problem illustrated on this figur is different from the one presented on figur 2 since the mean-line of each fibe is parallel to $e_{3}$. Moreover, in the following, we only use a 2-D model under plane strain assumption, this is why the problem treated in this paper corresponds to a microstructure composing of fiber depicted on figur 3 . Therefore, one fibe is represented by an ellipse parametrized by 5 constants: the horizontal and vertical positions $X$ and $Y$ of its center, an aspect ratio $\alpha$, the length of its semi-major axis $a$ and its orientation angle $\gamma$ according to vector $\boldsymbol{e}_{\mathbf{3}}$. Introducing the semi-minor axis $b$, the aspect ratio $\alpha$ is define by $\alpha=b / a$ with $\alpha \in[0,1]$. Using this geometrical parametrization, the probabilistic model is detailed in the following section.

\subsection{Stochastic modeling of the microstructure}

We firs introduce the numbers of fiber composing the microstructure denoted by $N_{f}$ which yields a number $N=5 N_{f}$ of geometrical parameters. However, if we generate exactly $N$ parameters representing $N_{f}$ fibers there is a high probability that several fiber intersect each other and thus do not verify the condition of impenetrability of the fibers detailed in Section 3.2.3 and which is equivalent to a rejection process. We then need to generate a greater number $n \gg N_{f}$ of "possible" geometrical parameters used in the rejection process. We here adopt a parametric approach to deal with the uncertainties. Let be $m=5 n$. The geometry is modeled by a $\mathbb{R}^{m}$ - valued random variable, define on a probability space $\left(\boldsymbol{\Xi}, \mathcal{B}_{\boldsymbol{\Xi}}, P_{\boldsymbol{\xi}}\right)$, whose support of the probability law $P_{\boldsymbol{\xi}}$ is $\boldsymbol{\Xi}$ which is a subset of $\mathbb{R}^{m}$. Considering the geometrical parametrization introduced in Section 3.1, the random 


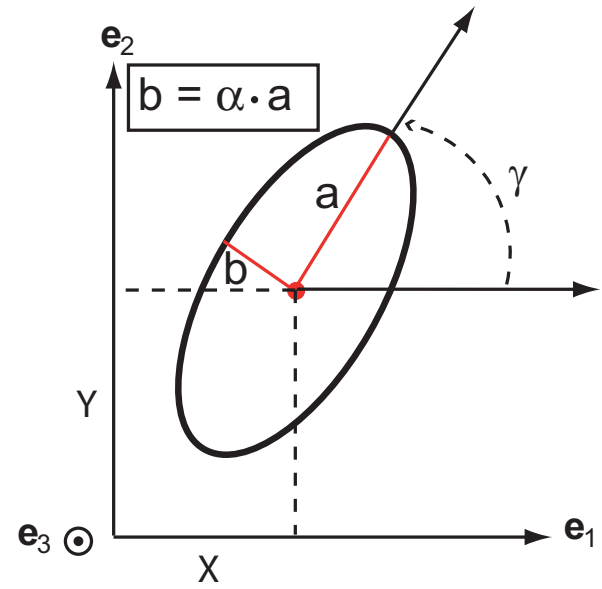

Figure 3. Geometrical description of a fibe

"possible" geometrical parameters is represented by the second-order random vector $\boldsymbol{\xi}$ define by

$$
\begin{aligned}
\boldsymbol{\xi} & =(\mathbf{X}, \mathbf{Y}, \boldsymbol{\alpha}, \mathbf{a}, \boldsymbol{\gamma}) \\
& =\left(X_{1}, \ldots, X_{n}, Y_{1}, \ldots, Y_{n}, \alpha_{1}, \ldots, \alpha_{n}, a_{1}, \ldots, a_{n}, \gamma_{1}, \ldots, \gamma_{n}\right) .
\end{aligned}
$$

The direct construction of $P_{\xi}$ is not a trivial task. We thus propose to construct a random generator associated with a statistical reduction method. Consequently, we construct $P_{\xi}$ using an indirect approach. The main steps of the development of this stochastic model via the construction of the random generator is given below.

1. Constructing a stochastic model of random vector $\boldsymbol{\xi}$ and generate a set of independent realizations $\left\{\boldsymbol{\xi}^{(k)}\right\}_{k=1}^{\nu}$ of $\boldsymbol{\xi}$, according to the parameters detailed in Section 3.2.1.

2. Performing a statistical reduction of random vector $\boldsymbol{\xi}$ using a principal component analysis yielding a new random vector $\boldsymbol{\zeta}=\left(\zeta_{1}, \ldots, \zeta_{M}\right)$ with $M \ll m$ and constructing the $\nu$ independent realizations (see Section 3.2.2).

3. Selecting the $\mu$ independent realizations $\left\{\boldsymbol{\zeta}^{(k)}\right\}_{k=1}^{\mu}$, with $\mu \leqslant \nu$, which verify the condition of impenetrability. These realizations completely characterize the stochastic model of the microstructure (see Section 3.2.3).

3.2.1. Stochastic model of $\boldsymbol{\xi}$ and random generator The aim is to generate the independent realizations $\left\{\boldsymbol{\xi}^{(k)}\right\}_{k=1}^{\nu}$ using the order statistics to impose the desired properties for the stochastic model. The random vectors $\mathbf{X}, \mathbf{Y}, \boldsymbol{\alpha}$, a and $\gamma$, yielding $\boldsymbol{\xi}$, are characterized by the independent realizations $\left\{\mathbf{X}^{(k)}\right\}_{k=1}^{\nu},\left\{\mathbf{Y}^{(k)}\right\}_{k=1}^{\nu},\left\{\boldsymbol{\alpha}^{(k)}\right\}_{k=1}^{\nu},\left\{\mathbf{a}^{(k)}\right\}_{k=1}^{\nu}$ and $\left\{\boldsymbol{\gamma}^{(k)}\right\}_{k=1}^{\nu}$. Since the realizations of each random vector $\mathbf{X}, \mathbf{Y}, \boldsymbol{\alpha}, \mathbf{a}$ and $\gamma$, will be generated through the same procedure, we present the methodology for random vector $\mathbf{X}$. To generate the realizations $\left\{\mathbf{X}^{(k)}\right\}_{k=1}^{\nu}$ with the desired properties, we introduce the order statistics. We then denote by $\mathbf{X}^{\text {ord }}=\left(X_{1}^{\text {ord }}, \ldots, X_{n}^{\text {ord }}\right)$ the random vector such that $X_{1}^{\text {ord, }(k)}<X_{2}^{\text {ord, }(k)}<\ldots<X_{n}^{\text {ord, }(k)}$ for each realization $k$ in $\{1, \ldots, \nu\}$. Let $\mathrm{E}\{\cdot\}$ be the mathematical expectation. In the construction proposed, the second-order quantities of the second-order random vector $\mathbf{X}^{\text {ord }}$ are controlled by the input parameters of the model, define as follows.

1. The mean vector $\mu_{\mathbf{X}^{\text {ord }}}=\mathrm{E}\left\{\mathbf{X}^{\text {ord }}\right\}$ whose components $\mu_{X_{1}^{\text {ord }}}, \ldots, \mu_{X_{n}^{\text {ord }}}$ are assumed to be equal to $\mu^{\text {ord }}=\mu_{X_{1}^{\text {ord }}}=\ldots=\mu_{X_{n}^{\text {ord }}}$.

2. The vector $\delta_{\mathbf{X}^{\text {ord }}}=\left(\delta_{X_{1}^{\text {ord }}}, \ldots, \delta_{X_{n}^{\text {ord }}}\right)$ where $\delta_{X_{i}^{\text {ord }}}$ is the coefficien of variation of random variable $X_{i}^{\text {ord }}$, is assumed to be such that $\delta^{\text {ord }}=\delta_{X_{1}^{\text {ord }}}=\ldots=\delta_{X_{n}^{\text {ord }}}$.

3. The spatial correlation is controlled by the positive real number $\rho$. 
Let $\left[\boldsymbol{C}_{\mathbf{X}^{\text {ord }}}\right]$ be the covariance matrix of random vector $\mathbf{X}^{\text {ord }}$ define by

$$
\left[\boldsymbol{C}_{\boldsymbol{X}^{\text {ord }}}\right]=\mathrm{E}\left\{\left(\mathbf{X}^{\text {ord }}-\boldsymbol{\mu}_{\mathbf{X}_{\text {ord }}}\right)\left(\mathbf{X}^{\text {ord }}-\boldsymbol{\mu}_{\mathbf{X}^{\text {ord }}}\right)^{T}\right\} .
$$

By construction of the stochastic model, the coefficient $\left[\boldsymbol{C}_{\mathbf{X}^{\text {ord }}}\right]_{i \ell}=\mathrm{E}\left\{\left(X_{i}^{\text {ord }}-\mu^{\text {ord }}\right)\left(X_{\ell}^{\text {ord }}-\mu^{\text {ord }}\right)\right\}$ are written as

$$
\begin{aligned}
& {\left[\boldsymbol{C}_{\boldsymbol{X}^{\text {ord }}}\right]_{i i}=1} \\
& {\left[\boldsymbol{C}_{\left.\boldsymbol{X}^{\text {ord }}\right]_{i \ell}}=\left(\delta^{\text {ord }}\right)^{2}\left(\mu^{\text {ord }}\right)^{2} \exp \left(\frac{|i-\ell|}{\rho n}\right) \text { if } i \neq \ell .\right.}
\end{aligned}
$$

We now introduce a random vector $\mathbf{U}=\left(U_{1}, \ldots, U_{n}\right)$ in which $U_{1}, \ldots, U_{n}$ are $n$ independent uniform random variables on [0 1]. Let $\left\{\mathbf{U}^{(k)}\right\}_{k=1}^{\nu}$ be $\nu$ independent realizations of $\mathbf{U}$.

For each realization $k$ in $\{1, \ldots, \nu\}$, we proceed as follows.

1. The components $U_{1}^{(k)}, \ldots, U_{n}^{(k)}$ of vector $\mathbf{U}^{(k)}$ are ordered according to the Euclidean distance $\left|U_{1}^{(k)}-U_{i}^{(k)}\right|$ for $i$ in $\{2, \ldots, n\}$. The resulting non-Gaussian random vector is denoted by $\mathbf{V}^{(k)}$.

2. We compute the vector $\widetilde{\mathbf{X}}^{(k)}=\boldsymbol{\mu}_{\mathbf{X} \text { ord }}+[\boldsymbol{L}]^{T} \mathbf{V}^{(k)}$ in which the matrix $[\boldsymbol{L}]$ is the upper triangular matrix coming from the Cholesky factorization $\left[\boldsymbol{C}_{\mathbf{X}^{\text {ord }}}\right]=[\boldsymbol{L}]^{T}[\boldsymbol{L}]$.

3. The targeted vector $\mathbf{X}^{(k)}$ is then obtained by ordering the vector $\widetilde{\mathbf{X}}^{(k)}$. One should notice that, since $\mathbf{V}^{(k)}$ is a non-Gaussian random vector, the Cholesky factorization implies that $\mathbf{X}^{(k)}$ is a non-Gaussian random vector.

By applying the above procedure to the $\nu$ realizations, we finall obtained $\left\{\mathbf{X}^{\text {ord, }(k)}\right\}_{k=1}^{\nu}$. The same methodology, with different or identical input parameters, is used to generate the independent realizations of the others random vectors $\mathbf{Y}, \alpha$, a and $\gamma$, and finall, to obtain the random vector $\boldsymbol{\xi}$.

In practice, since dimension $m$ of random vector $\boldsymbol{\xi}$ is high, it is not possible to directly use $\boldsymbol{\xi}$ in a parametric probabilistic approach. This difficult is circumvented in introducing a statistical reduction using a principal component analysis [35] of random vector $\boldsymbol{\xi}$.

3.2.2. Statistical reduction Let $\boldsymbol{\mu}_{\boldsymbol{\xi}}$ be the mean value of $\boldsymbol{\xi}$, estimated by $\boldsymbol{\mu}_{\boldsymbol{\xi}} \simeq \frac{1}{\nu} \sum_{k=1}^{\nu} \boldsymbol{\xi}^{(k)}$. Let us denote by $\widetilde{\boldsymbol{\xi}}^{(k)}=\boldsymbol{\xi}^{(k)}-\boldsymbol{\mu}_{\boldsymbol{\xi}}$ the centered realizations. The covariance matrix of random vector $\boldsymbol{\xi}$ is then estimated by

$$
\left[\boldsymbol{C}_{\boldsymbol{\xi}}\right] \simeq \frac{1}{(\nu-1)} \sum_{k=1}^{\nu} \widetilde{\boldsymbol{\xi}}^{(k)} \widetilde{\boldsymbol{\xi}}^{(k) T} .
$$

Let be $M \leq m$. Let $\lambda_{1} \geq \lambda_{2} \geq \ldots \geq \lambda_{M}$ and $\varphi_{1}, \varphi_{2}, \ldots, \varphi_{M}$ be the $M$ firs largest eigenvalues and the associated eigenvectors, of symmetric positive matrix $\left[C_{\xi}\right]$, such that $\left[C_{\xi}\right] \varphi_{j}=\lambda_{j} \varphi_{j}$. Vectors $\varphi_{1}, \ldots, \varphi_{M}$ are orthonormal. Using the principal component analysis, the mean-square approximation of $\boldsymbol{\xi}$ can be written as $\boldsymbol{\xi} \simeq \boldsymbol{\mu}_{\boldsymbol{\xi}}+\sum_{j=1}^{M} \sqrt{\lambda_{j}} \boldsymbol{\varphi}_{j} \zeta_{j}$ for which the second-order random variables $\zeta_{1}, \ldots, \zeta_{M}$ are centered and orthonormal, that is to say, $\mathrm{E}\left\{\zeta_{j}\right\}=0$ and $\mathrm{E}\left\{\zeta_{j} \zeta_{j^{\prime}}\right\}=\delta_{j j^{\prime}}$. The integer $M$ is assumed to be sufficientl large to reach the mean-square convergence which is controlled by

$$
\sqrt{1-\frac{\sum_{j=1}^{M} \lambda_{j}}{\operatorname{tr}\left[\boldsymbol{C}_{\boldsymbol{\xi}}\right]}}=\epsilon_{K L}^{M}<\epsilon_{K L},
$$

in which $\epsilon_{K L}$ is the tolerance parameter. The random variable $\zeta_{j}$ is given by $\zeta_{j}=\frac{1}{\sqrt{\lambda_{j}}} \boldsymbol{\varphi}_{j}^{T}\left(\boldsymbol{\xi}-\boldsymbol{\mu}_{\boldsymbol{\xi}}\right)$ and we introduce the random vector $\zeta=\left(\zeta_{1}, \ldots, \zeta_{M}\right)$. From these equations, it can be deduced the 
representation of the realizations $\boldsymbol{\xi}^{(k)}$ of $\boldsymbol{\xi}$ which are such that, for all $k=1, \ldots \nu$,

$$
\boldsymbol{\xi}^{(k)} \simeq \boldsymbol{\mu}_{\boldsymbol{\xi}}+\sum_{j=1}^{M} \sqrt{\lambda_{j}} \boldsymbol{\varphi}_{j} \zeta_{j}^{(k)}
$$

and the realization $\zeta_{j}^{(k)}$ of $\zeta_{j}$ is given by

$$
\zeta_{j}^{(k)}=\frac{1}{\sqrt{\lambda_{j}}} \boldsymbol{\varphi}_{j}^{T}\left(\boldsymbol{\xi}^{(k)}-\boldsymbol{\mu}_{\boldsymbol{\xi}}\right)
$$

Introducing the vector $\boldsymbol{\zeta}^{(k)}=\left(\zeta_{1}^{(k)}, \ldots, \zeta_{M}^{(k)}\right)$, we deduce that $\left\{\boldsymbol{\zeta}^{(k)}\right\}_{k=1}^{\nu}$ are the independent realizations of random vector $\zeta$ which represents the geometrical parameters of the fiber composing the microstructure.

3.2.3. Condition of impenetrability The fina step for obtaining the geometry of the microstructure consists in a selection of the realizations verifying the condition of impenetrability of the fiber which are characterized by domains $\Omega^{(r)}$ with $r=1, \ldots, N_{f}$. The condition of impenetrability is written as

$$
\bigcap_{r=1}^{N_{f}} \Omega^{(r)}=\emptyset .
$$

We will say that the condition of impenetrability is satisfie for a particular realization $\zeta^{(k)}$, if there exists a set of domains $\left\{\Omega^{(r)}\right\}_{r=1}^{N_{f}}$ that verifie equation (25). Each realization of the set $\left\{\boldsymbol{\zeta}^{(k)}\right\}_{k=1}^{\nu}$ of realizations is tested. If the condition of impenetrability is satisfied the realization is kept, otherwise it is rejected. With this procedure of rejection, we obtain the new set $\left\{\boldsymbol{\zeta}^{(k)}\right\}_{k=1}^{\mu}$ with $\mu \leqslant \nu$. In practice, the condition of impenetrability is verifie through a numerical procedure based on a discretization of the elementary cell define on domain $\Omega$ into a regular grid of points $\left\{\boldsymbol{x}_{i}\right\}_{i=1}^{N_{\Omega}}$ where $N_{\Omega}$ is taken sufficientl high to ensure that, at least, one point $\boldsymbol{x}_{i}$ is contained in each domain $\Omega^{(r)}$. The algorithm used to create the geometry of the microstructure acts as a rejection/acceptation algorithm: the possible fiber are tested one after another (a fibe is rejected or accepted if it does not intersect any existing fiber and stops when the desired number of fiber $N_{f}$ is reached.

3.2.4. Study of the probabilistic model Among the various input parameters of the stochastic model, detailed in section 3.2.1, the correlation parameter $\rho$ plays a major role in the efficien y of the reduction define by equation (23). Figure 4 illustrates the convergence of error $\epsilon_{K L}^{M}$ with respect to the number $M$ of eigenvalues for several values of $\rho$ equal to $0.1,0.3,0.5,0.7$ and 0.9 while others input parameters are fi ed to $m=2500, \nu=5000, \mu^{\text {ord }}=0.5$ and $\delta^{\text {ord }}=0.5$. We can observe a fast decrease of error $\epsilon_{K L}^{M}$ according to $M$ for any value of $\rho$. As expected, the fastest convergence is reached for the highest value of $\rho$ but the reduction is also effective for the lower values of $\rho$. However, even for the case $\rho=0.9$, we can notice that, at least, 9 eigenvalues are required to reach an error inferior to 0.1 . Figure 5 displays the graph of the probability density functions for several random variables $\zeta_{j}$ according to different values of the spatial correlation parameter $\rho$. We can observe that, even though the supports of the probability density functions of random variables $\zeta_{j}$ are essentially the same, the shape of the probability density function is different from a variable to another. The same remark can be pointed out when the spatial correlation parameter varies. It can even be seen, for particular values of $\rho$, a multimodal probability density function for particular random variable $\zeta_{j}$ (see for instance $\zeta_{4}$ with $\rho=0.5$ ).

As a fina illustration of the stochastic model, figur 6 displays some realizations of the random geometry of the microstructure for different values of input parameters.

3.2.5. Remark on the resulting random fiel In the fina part of this section, we study the properties of the random fiel generated by the proposed stochastic model. In the fiel of homogenization, 


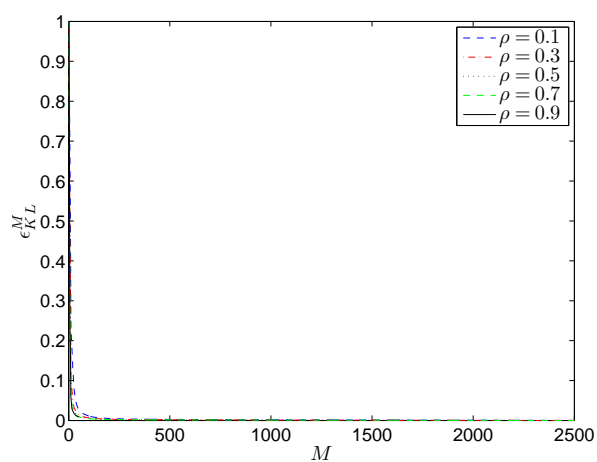

(a) $M \in\{1, \ldots, 2500\}$.

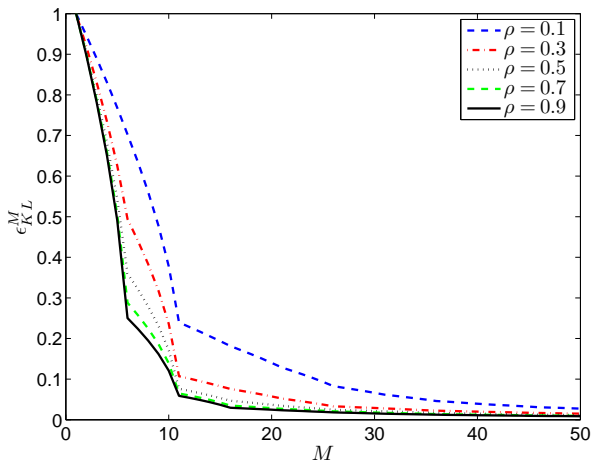

(b) $M \in\{1, \ldots, 50\}$.

Figure 4. Convergence of $\epsilon_{K L}^{M}$ according to the number $M$ of eigenvalues for different values of $\rho$ equal to $0.1,0.3,0.5,0.7$ and 0.9 .

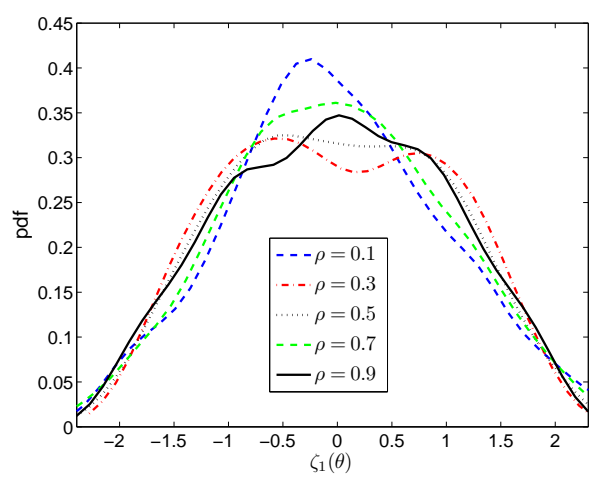

(a) Pdf of $\zeta_{1}$.

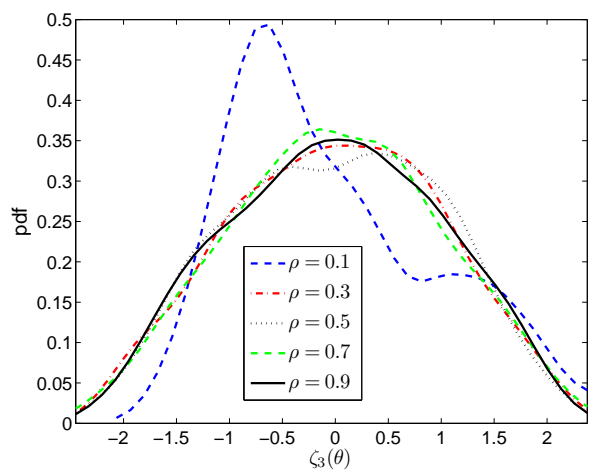

(c) Pdf of $\zeta_{3}$.

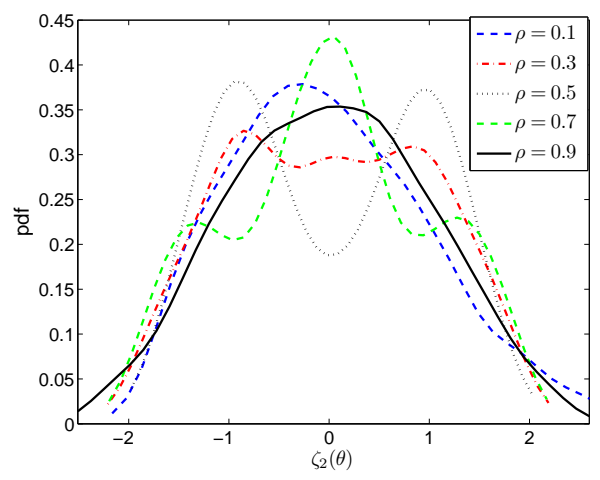

(b) Pdf of $\zeta_{2}$.

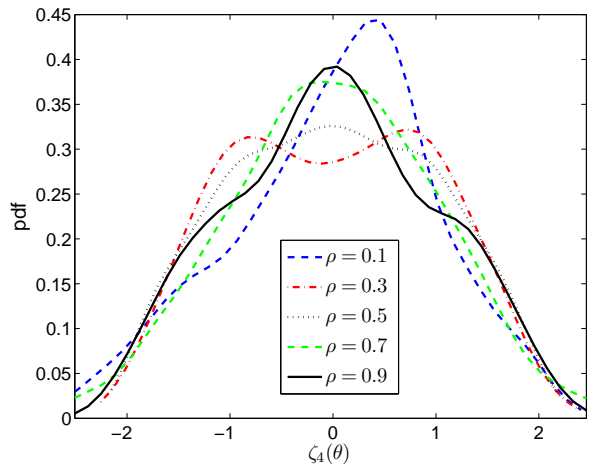

(d) Pdf of $\zeta_{4}$.

Figure 5. probability density functions of random variables $\zeta_{j}$ according to spatial correlation parameter $\rho$.

a crucial information comes from the separation of the scales. If the spatial correlation lengths of the mechanical field in the microstructure are small enough with respect of the dimension of the structure, then the scales can be separated and then a stochastic homogenization can be performed. In such a case, the statistical fluctuation at the macroscale are negligible and the effective mechanical properties are deterministic. In the other case, the scales are not separable and the effective properties are random. It is then important to calculate the spatial correlation lengths of the random fiel $\boldsymbol{L}(\boldsymbol{X})$ at the microscale. Thus, we propose to focus on the random fiel $\boldsymbol{L}(\boldsymbol{X})$ indexed by $\mathbb{R}^{d}$ through two quantities. The firs one is the spatial mean $\mu_{\|\boldsymbol{L}(\boldsymbol{X})\|_{F}}$ of $\boldsymbol{L}(\boldsymbol{X})$ define 


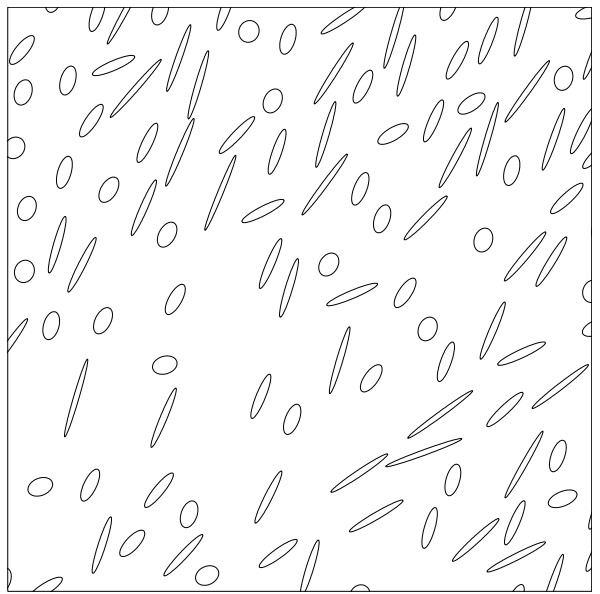

(a)

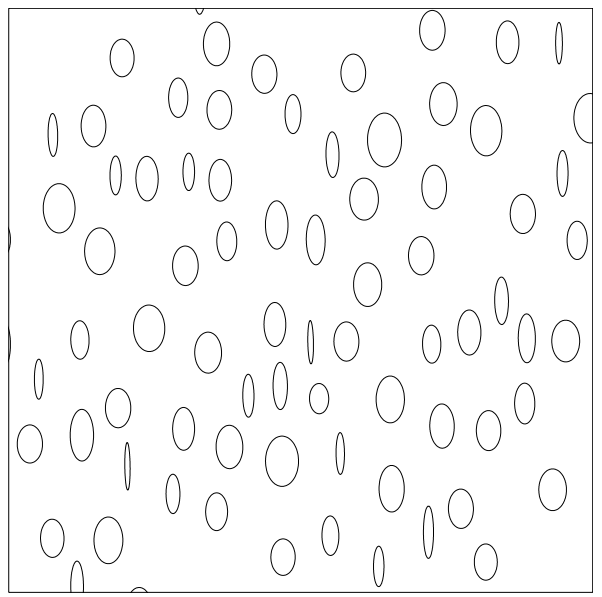

(c)

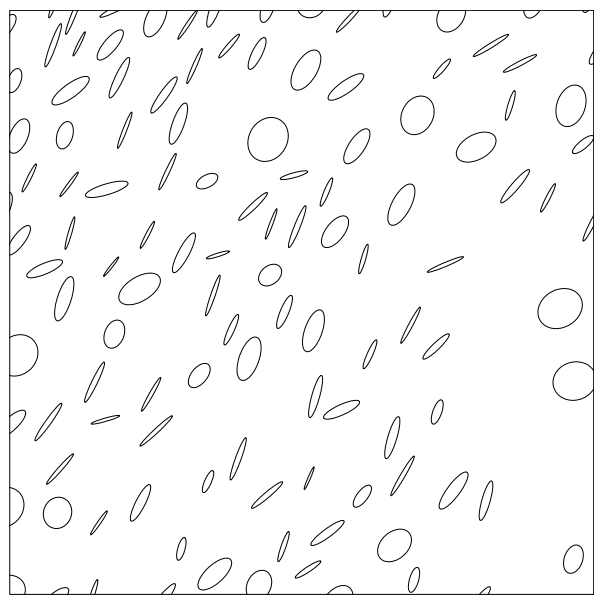

(e)

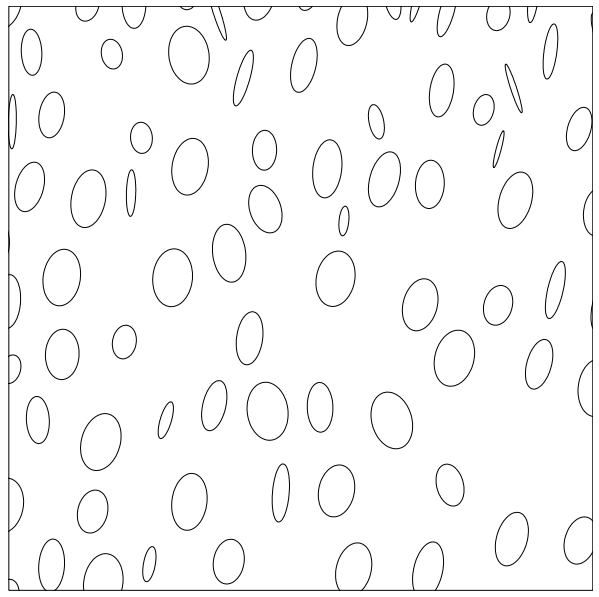

(b)

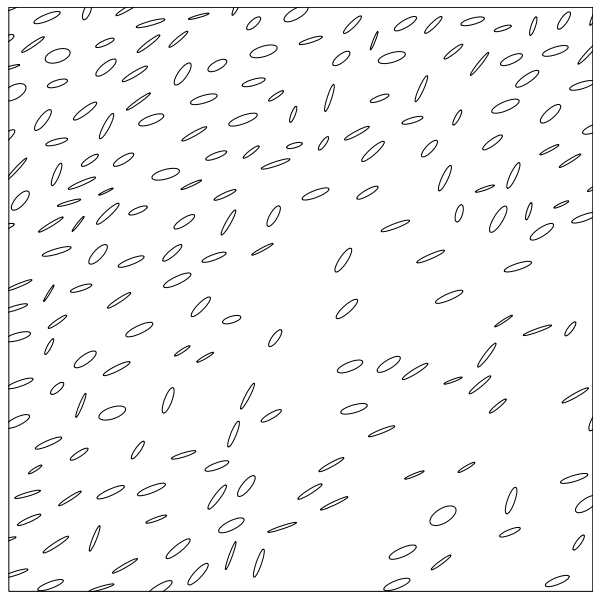

(d)

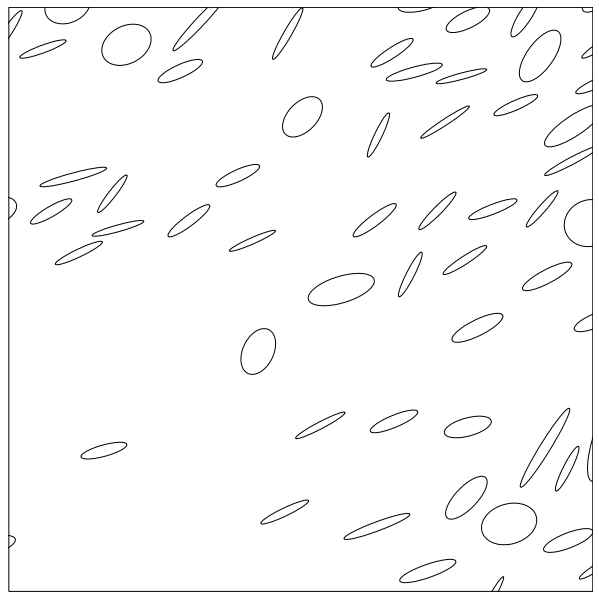

(f)

Figure 6. Various geometries of the random microstructure corresponding to different values of input parameters.

by $\mu_{\|\boldsymbol{L}(\boldsymbol{X})\|_{F}}=\mathrm{E}\left\{\|L(\boldsymbol{X})\|_{F}\right\}$ where $\|\cdot\|_{F}$ denotes the Frobenius norm. The second one is the 


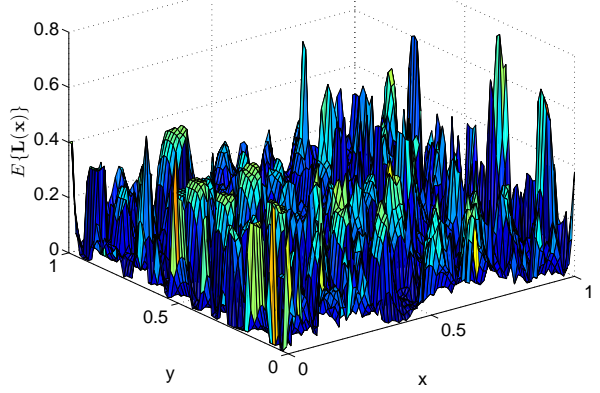

(a) Mean function $\boldsymbol{X} \mapsto \mu_{\|\boldsymbol{L}(\boldsymbol{X})\|_{F}}$.

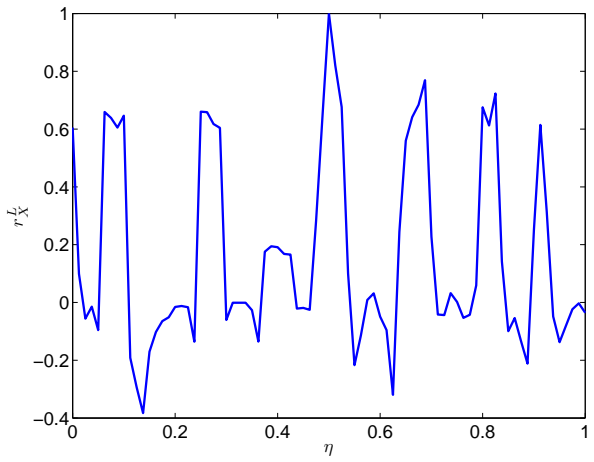

(b) Correlation function $\boldsymbol{\eta} \mapsto r_{L}(\boldsymbol{X}+\boldsymbol{\eta}, \boldsymbol{X})$ for $\boldsymbol{X}=(0.5,0.5)$.

Figure 7. Mean and correlation function of random fiel $\boldsymbol{L}(\boldsymbol{X})$.

correlation function $r_{L}(\boldsymbol{X}+\boldsymbol{\eta}, \boldsymbol{X})$ of $\boldsymbol{L}(\boldsymbol{X})$ define by

$$
r_{L}(\boldsymbol{X}+\boldsymbol{\eta}, \boldsymbol{X})=\frac{\left.\operatorname{tr}\left\{\mathrm{E}\{(\boldsymbol{L}(\boldsymbol{X}+\boldsymbol{\eta})-\mathrm{E}\{\boldsymbol{L}(\boldsymbol{X}+\boldsymbol{\eta})\})(\boldsymbol{L}(\boldsymbol{X})-\mathrm{E}\{\boldsymbol{L}(\boldsymbol{X}))\})^{T}\right\}\right\}}{\sqrt{\mathrm{E}\left\{\|\boldsymbol{L}(\boldsymbol{X}+\boldsymbol{\eta})-\mathrm{E}\{\boldsymbol{L}(\boldsymbol{X}+\boldsymbol{\eta})\}\|_{F}^{2}\right\} \mathrm{E}\left\{\|\boldsymbol{L}(\boldsymbol{X})-\mathrm{E}\{\boldsymbol{L}(\boldsymbol{X})\}\|_{F}^{2}\right\}}} .
$$

Figures 7(a) and 7(b) display the graphs of function $\boldsymbol{X} \mapsto \mu_{\|\boldsymbol{L}(\boldsymbol{X})\|_{F}}$ and $\boldsymbol{\eta} \mapsto r_{L}(\boldsymbol{X}+\boldsymbol{\eta}, \boldsymbol{X})$. If the two scales are separated then $\boldsymbol{L}(\boldsymbol{X})$ is a mean-square homogeneous second-order random fiel which involves that $\mu_{\|\boldsymbol{L}(\boldsymbol{X})\|_{F}}$ and $r_{L}(\boldsymbol{X}+\boldsymbol{\eta}, \boldsymbol{X})$ are independent of $\boldsymbol{X}$. However, figure 7(a) and 7 (b) clearly show that $\boldsymbol{L}(\boldsymbol{X})$ cannot be considered as a homogeneous random fiel on the elementary cell. Using equation (26) and using an exponential function to fi the correlation length $L$, we have estimated that $L_{R E V}=7$. That is to say that the REV associated to this specifi problem should be approximatively 7 times longer than the length of the elementary unit cell used in the manuscript where the geometrical characteristics of the fiber remain the same. Only the number $N_{f}$ should be increased to $N_{f}=7^{2} \cdot 80=3920$. Thus, we will see in the numerical example 5.1 that the macroscopic mechanical field have significan stochastic fluctuations

\section{METHOD OF STOCHASTIC NUMERICAL EXPLICIT POTENTIALS (S-NEXP)}

\subsection{Summary of the method}

The aim of the S-NEXP method is to numerically determine the strain density energy function $\bar{\psi}(\overline{\boldsymbol{C}})$ for a finit set of macroscopic boundary conditions depending on tensor $\overline{\boldsymbol{C}}$ (see equation (15)) and the different realizations of the random geometries of the microstructure define by the realizations $\left\{\boldsymbol{\zeta}^{(k)}\right\}_{k=1}^{\mu}$. The resulting values are then interpolated according to $\overline{\boldsymbol{C}}$ in order to obtain the components of tensors $\bar{S}$ and $\overline{\boldsymbol{L}}$ required to solve the macroscopic problem (16). The main steps of the approach are define as follows.

1. Definin the parameters domain $\Delta=\Delta_{\bar{C}} \times \Delta_{\zeta}$, where the domain $\Delta_{\bar{C}}$ is related to the components of the right Cauchy-Green strain tensor $\bar{C}$, and where the domain $\Delta_{\zeta}$ is related to the random geometrical parameters generated from the vector $\zeta$.

2. Discretizing each domain $\Delta_{\bar{C}}$ and $\Delta_{\zeta}$ into a finit number of nodes. For each node, the corresponding values of $\bar{C}$ are used to prescribe boundary conditions on the elementary cell and the corresponding values of $\zeta$ are used to defin the geometry of the microstructure. For each node the strain energy density function $\bar{\psi}(\bar{C}, \zeta)$ is determined numerically by solving problem (14). The results are stored in a database.

3. Giving the resulting database, a continuous interpolation of the discrete values is realized by an appropriate interpolation scheme associated with a tensorial representation of 
$\bar{\psi}(\bar{C}, \boldsymbol{\zeta})$. Then during the macroscopic finit element computations, the effective stressstrain relationship as well as the tangent matrix can be computed by deriving the continuous interpolation.

\subsection{Preliminaries}

In this work, for the sake of simplicity in the equations, we adopt the following notations, similar to the Voigt ones, to express the components of tensor $\bar{C}$. Let $\left\{\boldsymbol{e}_{1}, \boldsymbol{e}_{2}, \boldsymbol{e}_{3}\right\}$ be an orthonormal basis, then

$$
\begin{aligned}
\bar{C}= & \bar{C}_{1} e_{1} \otimes e_{1}+\bar{C}_{2} e_{2} \otimes e_{2}+\bar{C}_{3} e_{3} \otimes e_{3}+\frac{\bar{C}_{4}}{\sqrt{2}}\left(e_{2} \otimes e_{3}+e_{3} \otimes e_{2}\right) \\
+ & \frac{\bar{C}_{5}}{\sqrt{2}}\left(e_{1} \otimes e_{3}+e_{3} \otimes e_{1}\right)+\frac{\bar{C}_{6}}{\sqrt{2}}\left(e_{1} \otimes e_{2}+e_{2} \otimes e_{1}\right),
\end{aligned}
$$

yielding the following notations

$$
\bar{C}_{1}=\bar{C}_{11}, \quad \bar{C}_{2}=\bar{C}_{22}, \quad \bar{C}_{3}=\bar{C}_{33}, \quad \bar{C}_{4}=\sqrt{2} \bar{C}_{23}, \quad \bar{C}_{5}=\sqrt{2} \bar{C}_{13}, \quad \bar{C}_{6}=\sqrt{2} \bar{C}_{12} .
$$

In a similar manner, the components $\bar{S}_{\alpha}$, with $\alpha=1,2, \ldots, 6$, of tensor $\bar{S}$ and $L_{\alpha \beta}$, with $\beta=$ $1,2, \ldots, 6$, of tensor $\overline{\boldsymbol{L}}$ are such that

$$
\bar{S}_{\alpha}=2 \frac{\partial \bar{\psi}}{\partial \bar{C}_{\alpha}} \quad, \quad \bar{L}_{\alpha \beta}=4 \gamma_{\alpha \beta} \frac{\partial^{2} \bar{\psi}}{\partial \bar{C}_{\alpha} \partial \bar{C}_{\beta}}
$$

where $\gamma_{\alpha \beta}$ takes the values $1,1 / \sqrt{2}$ or $1 / 2$ (see [17] for the details).

\subsection{Computational details}

In this section, we described the main numerical aspects and references, required to implement the different steps of the method listed in Section 4.1.

4.3.1. Definitio of domain $\Delta$ The global parameters domain $\Delta$ is split into two domains such that

$$
\Delta=\Delta_{\bar{C}} \times \Delta_{\zeta} \quad \text { where } \quad\left\{\begin{array}{l}
\Delta_{\bar{C}}=\Delta_{\bar{C}_{1}} \times \Delta_{\bar{C}_{2}} \times \ldots \times \Delta_{\bar{C}_{D}} \\
\Delta_{\zeta}=\Delta_{\zeta_{1}} \times \Delta_{\zeta_{2}} \times \ldots \times \Delta_{\zeta_{M}}
\end{array} .\right.
$$

The firs issue is related to the definitio of domain $\Delta_{\bar{C}}$ and $\Delta_{\zeta}$. Extreme values $\Delta_{C_{i}}^{\text {min }}$ and $\Delta_{C_{i}}$ max of a domain $\Delta_{\bar{C}_{i}}$ can be arbitrarily chosen. However, this case may involve difficultie during the macroscopic computations since some strain states may correspond to values of $\bar{C}_{i}$ which are outside the domain $\Delta_{\bar{C}_{i}}$. To avoid this difficult, preliminary macroscopic simulations can be done with homogeneous behaviors corresponding to each phase of the microstructure. However, there is still a risk that a strain state falls outside the domain $\Delta_{\bar{C}_{i}}$. In that case, one may use the extrapolation technique given in [19] to avoid this problem. Thus, we can determine a priori the range of strains levels in the structure and fi the values of the extrema $\Delta \bar{C}_{i}$ min and $\Delta_{\bar{C}_{i}}^{\max }$. Extreme values $\Delta_{\zeta_{i}}^{\min }$ and $\Delta_{\zeta_{i}}^{\max }$ of a domain $\Delta_{\zeta_{i}}$ are naturally chosen using the set $\left\{\zeta_{i}^{(k)}\right\}_{k=1}^{\mu}$. A firs way consists in taking the extreme values of the variable $\zeta_{i}$ but this strategy may lead to a large range of variation for $\zeta_{i}$ and may require a fin discretization of the domain $\Delta_{\zeta_{i}}$ only needed for an accurate approximation of values of $\zeta_{i}$ corresponding to a low probability. Thus, we propose to defin the extreme values $\Delta_{\zeta_{i}}^{\min }$ and $\Delta_{\zeta_{i}}^{\max }$ on the quantile values (quantiles at $5 \%$ and $95 \%$ for instance) which allow reducing the support of the variable $\zeta_{i}$.

The second issue arises from the discretization of domains $\Delta_{\bar{C}}$ and $\Delta_{\zeta}$. Several ways are possible as non-uniform or uniform discretizations. In this work, we use the same discretization as proposed in $[17,19]$ yielding a uniform discretization. We choose a rectangular grid leading us to discretize each domain $\Delta_{\bar{C}_{i}}$ into a number $p$ of nodes homogeneously distributed. Then, the domain $\Delta_{\bar{C}}$ is 
discretized with $p^{D}$ nodes. Concerning the domains $\Delta_{\zeta_{i}}$ related to $\Delta_{\zeta}$, the same type of procedure is used. Each domain is discretized into a f xed number $q$ of nodes homogeneously distributed. It then yields a number $q^{M}$ of nodes for domain $\Delta_{\zeta}$. One can notice that a choice of discretization based on the quantile values is possible and appears to be a more sophisticated way in order to be consistent with the probabilistic content of the problem. This kind of non-homogeneous discretization has been investigated by the authors but has led us to poorer results than the homogeneous one mainly due to the high fluctuation of the solution. Thus the overall parameters domain $\Delta$ is discretized into a number $p^{D} \times q^{M}$ of nodes for which nonlinear FEM computations are required to compute the values of the strain energy density function $\psi(\overline{\boldsymbol{C}}, \boldsymbol{\zeta})$. The FEM computations are not performed independently: for one geometrical realization $\zeta^{(k)}$, we take as an initial guess for the NewtonRaphson algorithm, the solution, among the ones previously calculated, which is the "closest" to the one to be computed. Finally, the different values of $\psi(\overline{\boldsymbol{C}}, \boldsymbol{\zeta})$ are stored in a hypermatrix $\overline{\mathbb{W}}$ which represents the database.

4.3.2. Separated variables interpolation approach Since the hypermatrix $\overline{\mathbb{W}}$ is computed, we seek to obtain a continuous explicit form of the potential $\bar{\psi}$ according to tensor $\bar{C}$ and vector $\zeta$. We use the methodology proposed in [19] and we extend it to the stochastic framework. The two distinct steps are the following.

1. Computation of a separated variables representation $\overline{\mathbb{U}}$ of $\overline{\mathbb{W}}$ through a Parallel Factor Decomposition [37].

2. Computation of a continuous interpolation of $\overline{\mathbb{U}}$ using twice continuously differentiable $\left(\mathcal{C}^{2}\right)$ functions so that we can evaluate the components of tensors $\bar{S}$ and $\overline{\boldsymbol{L}}$.

The separated variables representation consists in decomposing $\overline{\mathbb{W}}$ into a tensorial representation $\overline{\mathbb{U}}$ such that

$$
\overline{\mathbb{W}} \approx \overline{\mathbb{U}}=\sum_{r=1}^{R} \bigotimes_{k_{1}=1}^{D} \phi_{k_{1}}^{r} \bigotimes_{k_{2}=1}^{M} \phi_{k_{2}}^{r},
$$

where $\left\{\boldsymbol{\phi}_{k_{1}}^{r}, k_{1}=1, \ldots, D\right\}$ are real-valued vectors related to the macroscopic strain tensor component $\bar{C}_{i}$, and where $\left\{\boldsymbol{\phi}_{k_{2}}^{r}, k_{2}=1, \ldots, M\right\}$ are real-valued vectors related to the geometrical parameters of the geometry and $R$ is an integer. For a given $R$, the vectors $\phi_{k_{1}}^{r}$ and $\phi_{k_{2}}^{r}$ can be constructed by solving the following least square problem,

$$
\underset{\phi_{k_{1}}^{r}, \phi_{k_{2}}^{r}}{\inf }\left\|\overline{\mathbb{W}}-\sum_{r=1}^{R} \bigotimes_{k_{1}=1}^{D} \phi_{k_{1}}^{r} \bigotimes_{k_{2}=1}^{M} \phi_{k_{2}}^{r}\right\|_{F}^{2} .
$$

Introducing a tolerance parameter $\delta_{\text {sep }}$ in order to reach the desired accuracy, integer $R$ is increased until

$$
\left\|\overline{\mathbb{W}}-\sum_{r=1}^{R} \bigotimes_{k_{1}=1}^{D} \phi_{k_{1}}^{r} \bigotimes_{k_{2}=1}^{M} \phi_{k_{2}}^{r}\right\|_{F}<\delta_{\text {sep }} .
$$

It should be noted that problem define by equation (33) is nonlinear according to the unknown vectors $\phi_{k_{1}}^{r}$ and $\phi_{k_{2}}^{r}$. Thus, we use an iterative procedure to solve the problem with the alternated

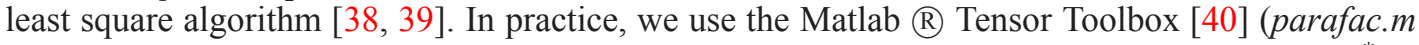
function) to numerically solve the problem. From equation (32), the continuous interpolation $\bar{\psi}^{*}$ of $\bar{\psi}$ can be obtained as

$$
\bar{\psi} \approx \bar{\psi}^{*}=\sum_{r=1}^{R} \prod_{k_{1}=1}^{D} \widetilde{\phi}_{k_{1}}^{r}\left(\bar{C}_{k_{1}}\right) \prod_{k_{2}=1}^{M} \widetilde{\phi}_{k_{2}}^{r}\left(\zeta_{k_{2}}\right),
$$


where $\widetilde{\phi}_{j}^{r}\left(\omega_{j}\right)$ are the interpolated values of $\phi_{j}^{r}$ define by

$$
\widetilde{\phi}_{j}^{r}\left(\omega_{j}\right)=\sum_{k=1}^{n} N_{k}\left(\omega_{j}\right)\left\{\phi_{j}^{r}\right\}_{k} .
$$

In equation (36), $N_{k}$ is one-dimensional $\mathcal{C}^{2}$ interpolation function associated with node $k, n$ denotes the number of nodes supporting the shape functions $N_{k}\left(\omega_{j}\right)$ and $\left\{\phi_{j}^{r}\right\}_{k}$ are the discrete values of $\phi_{j}^{r}$. Thus, the second Piola-Kirchhoff stress can be approximated by

$$
\bar{S}_{i}^{*}\left(\bar{C}_{1}, \ldots, \bar{C}_{D}, \zeta_{1}, \ldots, \zeta_{M}\right)=2 \sum_{r=1}^{R}\left(\left\{\prod_{k_{1} \neq i} \widetilde{\phi}_{k_{1}}^{r}\left(\bar{C}_{k_{1}}\right) \prod_{k_{2}} \widetilde{\phi}_{k_{2}}^{r}\left(\zeta_{k_{2}}\right)\right\} \frac{\partial \widetilde{\phi}_{i}^{r}\left(\bar{C}_{i}\right)}{\partial \bar{C}_{i}}\right),
$$

in which $k_{1}=1, \ldots, D$ and $k_{2}=1, \ldots, M$. In the same manner, the approximated value $\overline{\boldsymbol{L}}^{*}$ of tangent elastic tensor $\overline{\boldsymbol{L}}$ is given by

$$
\begin{aligned}
& \bar{L}_{i j}^{*}=4 \gamma_{i j} \sum_{r=1}^{R}\left(\left\{\prod_{k_{1} \neq i, j} \widetilde{\phi}_{k_{1}}^{r}\left(\bar{C}_{k_{1}}\right) \prod_{k_{2}} \widetilde{\phi}_{k_{2}}^{r}\left(\zeta_{k_{2}}\right)\right\} \frac{\partial \widetilde{\phi}_{i}^{r}\left(\bar{C}_{i}\right)}{\partial \bar{C}_{i}} \frac{\partial \widetilde{\phi}_{j}^{r}\left(\bar{C}_{j}\right)}{\partial \bar{C}_{j}}\right) \text { if } i \neq j, \\
& \bar{L}_{i i}^{*}=4 \gamma_{i i} \sum_{r=1}^{R}\left(\left\{\prod_{k_{1} \neq i} \widetilde{\phi}_{k_{1}}^{r}\left(\bar{C}_{k_{1}}\right) \prod_{k_{2}} \widetilde{\phi}_{k_{2}}^{r}\left(\zeta_{k_{2}}\right)\right\} \frac{\partial^{2} \widetilde{\phi}_{i}^{r}\left(\bar{C}_{i}\right)}{\partial \bar{C}_{i}^{2}}\right),
\end{aligned}
$$

with $k_{1}=1, \ldots, D$ and $k_{2}=1, \ldots, M$ and with

$$
\frac{\partial \widetilde{\phi}_{i}^{r}\left(\bar{C}_{i}\right)}{\partial \bar{C}_{i}}=\sum_{k=1}^{n} \frac{\partial N_{k}\left(\bar{C}_{i}\right)}{\partial \bar{C}_{i}}\left\{\phi_{i}^{r}\right\}_{k} \quad \text { and } \quad \frac{\partial^{2} \widetilde{\phi}_{i}^{r}\left(\bar{C}_{i}\right)}{\partial \bar{C}_{i}^{2}}=\sum_{k=1}^{n} \frac{\partial^{2} N_{k}\left(\bar{C}_{i}\right)}{\partial \bar{C}_{i}^{2}}\left\{\phi_{i}^{r}\right\}_{k} .
$$

Many choices are possible for the interpolation functions $N_{k}$. In this work, we use one-dimensional $\mathcal{C}^{2}$ cubic spline functions as in $[17,19]$ for which results of convergence with respect to the number of discretization points have been studied (see for instance [41]). For a parameters domain of high dimension, this approach has the advantage to only determine the coefficient of onedimensional spline functions and then leads us to solve a small system of equations, which saves both computational time and memory.

\section{NUMERICAL EXAMPLES}

Two numerical examples are developed in this section. The firs one aims at demonstrating the efficien y of the proposed method for the approximation of the macroscopic constitutive behavior through tensors $\overline{\boldsymbol{S}}(\overline{\boldsymbol{C}}, \boldsymbol{\zeta})$ and $\overline{\boldsymbol{L}}(\overline{\boldsymbol{C}}, \boldsymbol{\zeta})$. The numerical potential $\bar{\psi}(\overline{\boldsymbol{C}}, \boldsymbol{\zeta})$ obtained in the firs example is then used in the second example which deals with a macroscopic problem. For both problems, solutions are compared with reference solutions computed with classical methods.

\subsection{Numerical example 1: study of the microscopic problem}

5.1.1. Problem definitio The aim of this firs example is to demonstrate the efficien y of the S-NEXP method and the accuracy of the resulting solution through comparisons with standard approaches (nonlinear FEM computations and Monte-Carlo simulations). For this purpose, we focus on the quantities $\bar{\psi}\left(\bar{C}_{1}, \ldots, \bar{C}_{D} ; \zeta_{1}, \ldots, \zeta_{M}\right)$ and $\bar{S}_{i}\left(\bar{C}_{1}, \ldots, \bar{C}_{D} ; \zeta_{1}, \ldots \zeta_{M}\right)$ according to macroscopic strains $\bar{C}$ and reduced random variables $\zeta$ characterizing the random geometry of the microstructure. The parameters of the model are the following ones:

1. Random generator parameters: number of random variables $m=6000$, spatial correlation parameter $\rho=0.5$, number of realizations $\nu=1000$, coefficien of variation $\delta^{\text {ord }}=0.5$ and number of fiber $N_{f}=80$. 
2. Statistical reduction (23): we have chosen $M=2$ eigenvalues in order to facilitate the understanding for the response surfaces corresponding to $\epsilon_{K L}^{1}=0.8$ and $\epsilon_{K L}^{2}=0.7$. The following values of the tolerance parameter are $\epsilon_{K L}^{3}=0.55, \epsilon_{K L}^{4}=0.38$ and $\epsilon_{K L}^{5}=0.15$.

3. Discretization of domain $\Delta_{\bar{C}}$ : the macroscopic strain domain is chosen such as $\Delta_{\bar{C}}=\Delta_{\bar{C}_{1}} \times$ $\Delta_{\bar{C}_{2}} \times \Delta_{\bar{C}_{6}}=[1,1.5] \times[1,1.5] \times[0,0.5]$. We use $p=5$ points for each domain $\Delta_{\bar{C}_{i}} . \mathrm{A}$ convergence analysis of the deterministic problem only related to the macroscopic strains can be found in [19].

4. Material parameters: we use a compressible Neo-Hookean model given by the potential define by equation (5). For the matrix, the Young modulus is $E^{m}=10^{3} \mathrm{MPa}$ and the Poisson ratio is $\nu^{m}=0.4$. For the inclusions, $E^{f}=10^{5} \mathrm{MPa}$ and $\nu^{f}=0.3$.

In this example, we firs focus on the efficien y of the method according to the number $q$ of points used for the discretization of parameters domain $\Delta_{\zeta}$. Here, the domain $\Delta_{\zeta}=\Delta_{\zeta_{1}} \times \Delta_{\zeta_{2}}=$ $\left[q_{5}^{1}, q_{95}^{1}\right] \times\left[q_{5}^{2}, q_{95}^{2}\right]=[-1.61,1.63] \times[-1.7,1.55]$ where $q_{5}^{i}$ and $q_{95}^{i}$ are respectively the quantile values at $5 \%$ and $95 \%$ for the $i$-th random variable. We choose to discretize each domain $\Delta_{\zeta_{i}}$ with different numbers $q=5,11$ and 21 of points homogeneously distributed.

5.1.2. Influenc of random variable $\zeta_{1}$ In this section, we study the influenc of random variable $\zeta_{1}$ on the S-NEXP solution and the resulting macroscopic constitutive behavior through the second Piola-Kirchhoff stress tensor $\overline{\boldsymbol{S}}$. Figure 8 illustrates the response surfaces of $\bar{\psi}(\overline{\boldsymbol{C}}, \boldsymbol{\zeta})$, according to $\zeta_{1}$ (and for $\zeta_{2}$ fi ed to zero) and to macroscopic strains such that $\left(\bar{C}_{11}, \bar{C}_{22}, \bar{C}_{12}\right)$ belongs to $[1,1.5] \times[1,1.5] \times[0,0.5]$, for the three choices of discretization of $\Delta_{\zeta}$. Figure 8 also displays a reference solution consisting in a set of direct nonlinear FEM computations for a given grid of $20 \times 20$ points. One can observe a good convergence of the solution towards the reference solution with respect of number of discretization points $q$. The solution corresponding to $q=21$ is obviously the one which matches the best the reference solution. Figure 9 presents the response surfaces related to component $\bar{S}_{11}(\overline{\boldsymbol{C}}, \boldsymbol{\zeta})$ of tensor $\overline{\boldsymbol{S}}$ according to the same parameters as in figur 8 . We also notice a good convergence of the S-NEXP solution according to the number of discretization points $q$. It clearly shows that the numerical derivation of the potential $\bar{\psi}(\overline{\boldsymbol{C}}, \boldsymbol{\zeta})$ allows a good approximation of the macroscopic constitutive behavior. Moreover, one should notice that the post-processing of the S-NEXP solution is very fast thanks to the explicit continuous form of the S-NEXP solution.

In figure 8 and 9 , we can observe high variations of the quantities of interest according to parameter $\zeta_{1}$ which can be naturally related to the stochastic fluctuations However, with a quite high number of discretization points $(q=11$ and 21$)$, the S-NEXP method allows us to accurately capture irregularities in the solution. For instance, in figur 9, we can notice an abrupt variation of the solution around the value $\zeta_{1} \approx 0$. This is due to a significan change in the geometry as shown on figur 10 where two realizations of the geometry are displayed. The same variations appear in the components of the elastic tangent tensor $\overline{\boldsymbol{L}}(\overline{\boldsymbol{C}}, \boldsymbol{\zeta})$ as it is shown on figur 11 where the response surfaces of the component $\bar{L}_{1111}$ are displayed for the different S-NEXP solutions and the reference solution.

We then focus on the probability density functions of the previous quantities of interest for a particular macroscopic strain state corresponding to $\left\{\bar{C}_{11}=1.5 ; \bar{C}_{22}=1.5 ; \bar{C}_{12}=0.5\right\}$. We use the $\mu$ realizations $\zeta^{(k)}$ which verify the condition of impenetrability given in Section 3.2.3. As a reference solution, we use realizations of the geometry of the microstructure generated with the reduced-order model corresponding to $M=1$ for which we perform nonlinear FEM computations in order to obtain the reference values of $\bar{\psi}$ and $\bar{S}_{11}$. Figure 12 illustrates these probability density functions for the different chosen discretizations of parameters domain $\Delta_{\zeta}$ and for the reference solution. As for the response surfaces, we can observe a good convergence of the solution according to the number of discretization points $q$.

5.1.3. Influenc of random variables $\zeta_{1}$ and $\zeta_{2}$ In the previous section, we have noticed that the solution showed a significan variability according to $\zeta_{1}$. We now focus on the influenc of both random variables $\zeta_{1}$ and $\zeta_{2}$ on the solution. Figures 13 and 14 illustrate respectively the response surfaces of $\bar{\psi}$ and $\bar{S}_{11}$ according to $\zeta_{1}$ and $\zeta_{2}$ for the S-NEXP solution (with $q=21$ ) and the 


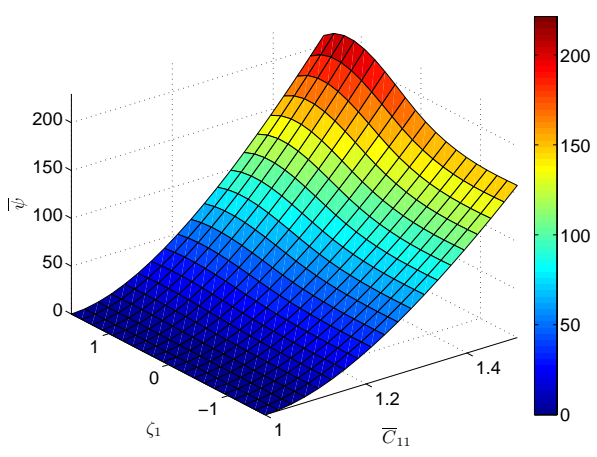

(a) S-NEXP response surface of $\bar{\psi}$ for $q=5$.

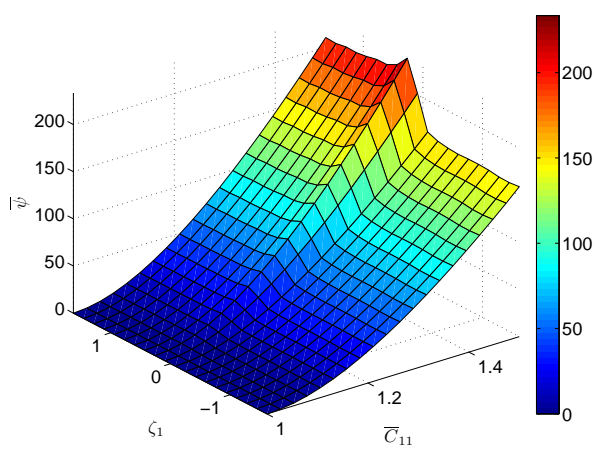

(c) S-NEXP response surface of $\bar{\psi}$ for $q=21$.

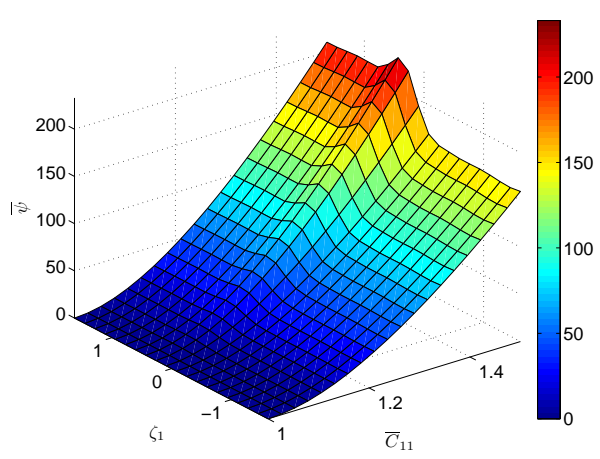

(b) S-NEXP response surface of $\bar{\psi}$ for $q=11$.

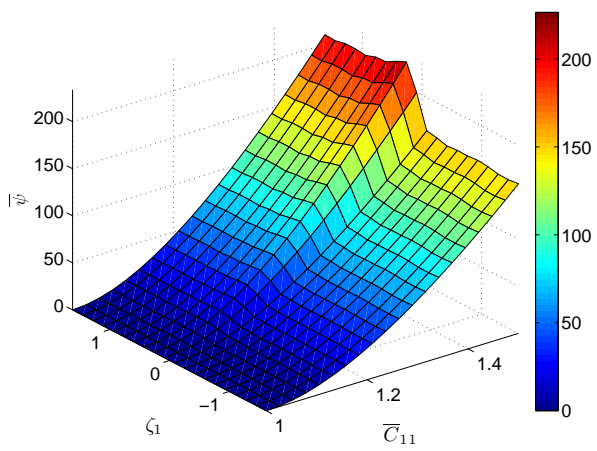

(d) Reference response surface of $\bar{\psi}$.

Figure 8. Response surfaces of $\bar{\psi}$ according to $\zeta_{1}$ and macroscopic strains such that $\left(\bar{C}_{11}, \bar{C}_{22}, \bar{C}_{12}\right)$ belongs to $[1,1.5] \times[1,1.5] \times[0,0.5]$ for $q=5, q=11$ and $q=21$ and for the reference solution.

reference solution (nonlinear FEM computations). On those two cases the macroscopic strain state corresponds to $\bar{C}_{11}=1.5, \bar{C}_{22}=1.5$ and $\bar{C}_{12}=0.5$. As expected, we can notice significan global and local variations which disturb the S-NEXP solution. However, the method leads us to acceptable results with respect to the reference solution. As explained in Section 5.1.2, the global variations are due to significan changes in the geometry while the local variations are mainly due to the numerical difficultie involved by problem define by equation (14) associated with boundary conditions define by equation (15).

Figure 15 illustrates the probability density functions of $\bar{\psi}$ and component $\bar{S}_{22}$ for the S-NEXP solution (with $q=11$ ) and the reference solution (based on the reduced-order model corresponding to $M=2$ ) corresponding to the macroscopic strain state $\bar{C}_{11}=1.5 \bar{C}_{22}=1.5$ and $\bar{C}_{12}=0.5$. We can observe slight differences between the two solutions. Nevertheless, the S-NEXP solution appears satisfying according to the reference solution.

5.1.4. Accuracy and computational costs of the S-NEXP approximation In this section we present a convergence analysis in order to estimate the accuracy of the S-NEXP approximation. As we have no a priori information on the probabilistic content of the macroscopic strains, we suppose that all components of stress tensors $\overline{\boldsymbol{S}}$ are independent uniform random variables such as $\bar{C}_{11} \sim \mathcal{U}(1,1.5)$, $\bar{C}_{22} \sim \mathcal{U}(1,1.5)$ and $\bar{C}_{12} \sim \mathcal{U}(0,0.5)$, in which $\mathcal{U}(a, b)$ denotes a uniform random variable on $[a, b]$. We can thus introduce an error indicator $\epsilon_{q}$ define as follows

$$
\epsilon_{q}=\frac{\left\|J_{q}-J_{r e f}\right\|_{L^{2}\left(\boldsymbol{\Xi}, d P_{\Xi}\right)}}{\left\|J_{r e f}\right\|_{L^{2}(\boldsymbol{\Xi}, d P \Xi)}},
$$




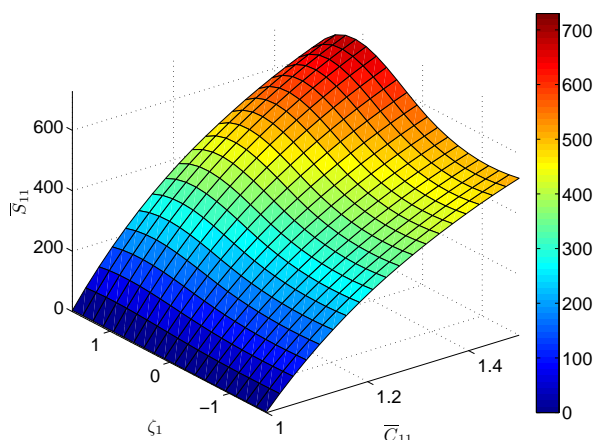

(a) S-NEXP response surface of $\bar{S}_{11}$ for $q=5$.

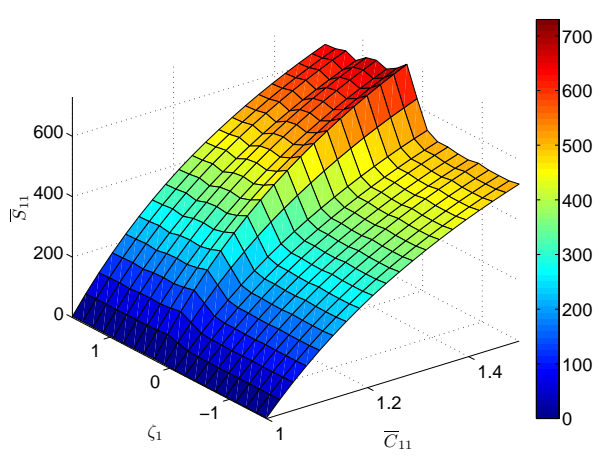

(c) S-NEXP response surface of $\bar{S}_{11}$ for $q=21$.

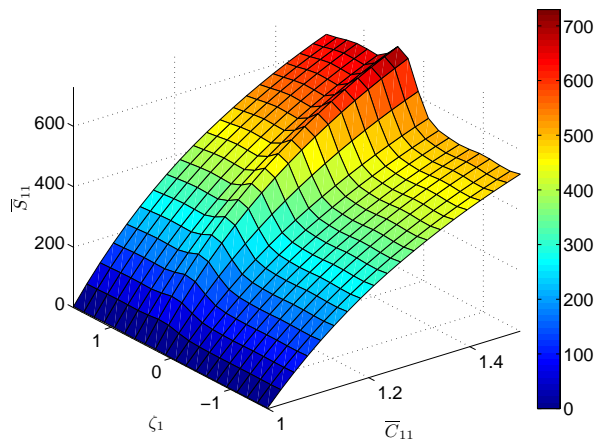

(b) S-NEXP response surface of $\bar{S}_{11}$ for $q=11$.

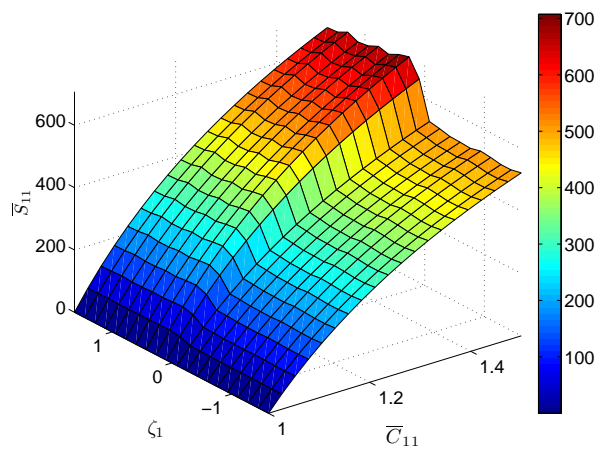

(d) Reference response surface of $\bar{S}_{11}$

Figure 9. Response surfaces of $\bar{S}_{11}$ according to $\zeta_{1}$ and macroscopic strains such that $\left(\bar{C}_{11}, \bar{C}_{22}, \bar{C}_{12}\right)$ belongs to $[1,1.5] \times[1,1.5] \times[0,0.5]$ for $q=5, q=11$ and $q=21$ and for the reference solution.

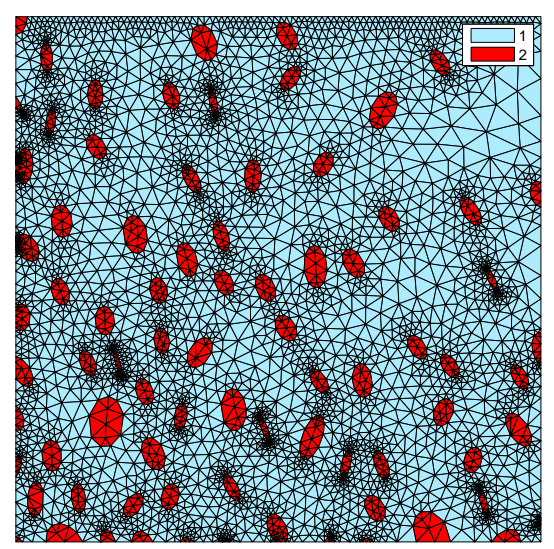

(a) Mesh of the microstructure corresponding to $\zeta_{1}^{(1)}=-0.32$.

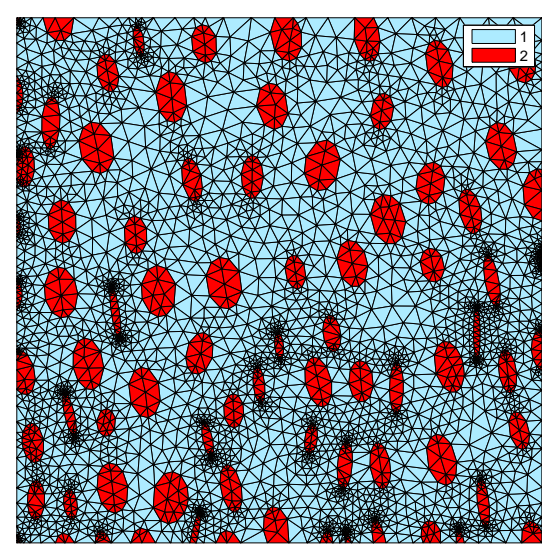

(b) Mesh of the microstructure corresponding to $\zeta_{1}^{(2)}=0.33$.

Figure 10. Geometries and meshes of the microstructure for two realizations of $\zeta_{1}$.

with

$$
\left\|J_{r e f}\right\|_{L^{2}\left(\Xi, d P_{\Xi}\right)} \simeq \frac{1}{\mu} \sum_{k=1}^{\mu}\left|J_{\text {ref }}^{(k)}\right|^{2},
$$




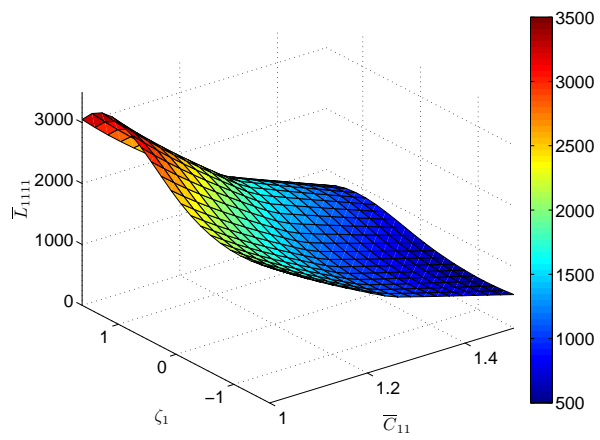

(a) S-NEXP response surface of $\bar{L}_{1111}$ for $q=5$.

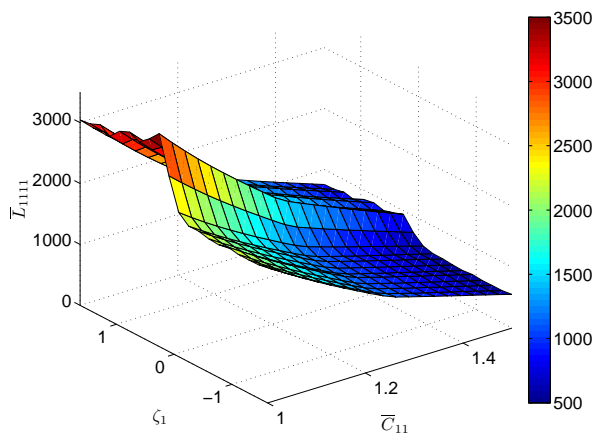

(c) S-NEXP response surface of $\bar{L}_{1111}$ for $q=21$.

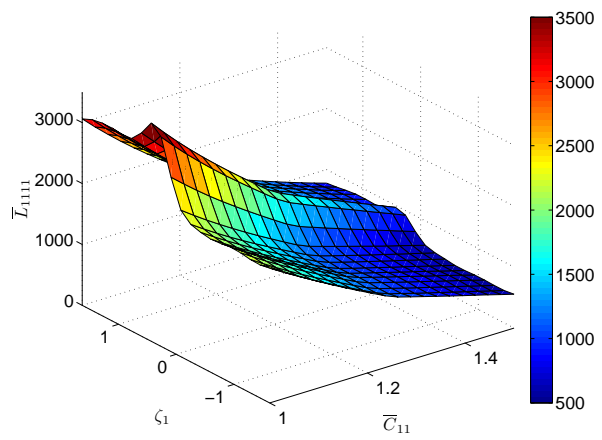

(b) S-NEXP response surface of $\bar{L}_{1111}$ for $q=11$.

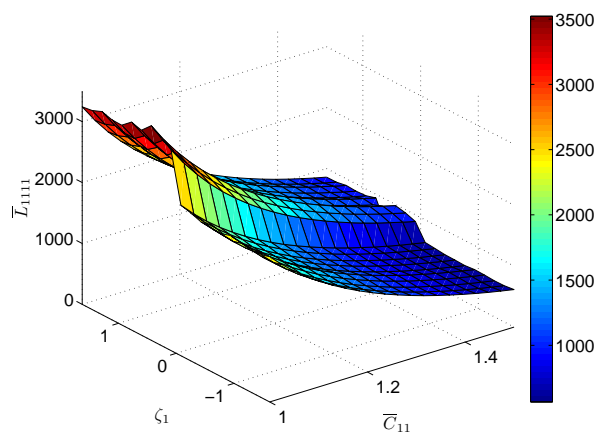

(d) Reference response surface of $\bar{L}_{1111}$

Figure 11. Response surfaces of $\bar{L}_{1111}$ according to $\zeta_{1}$ and macroscopic strains such that $\left(\bar{C}_{11}, \bar{C}_{22}, \bar{C}_{12}\right)$ belongs to $[1,1.5] \times[1,1.5] \times[0,0.5]$ for $q=5, q=11$ and $q=21$ and for the reference solution.

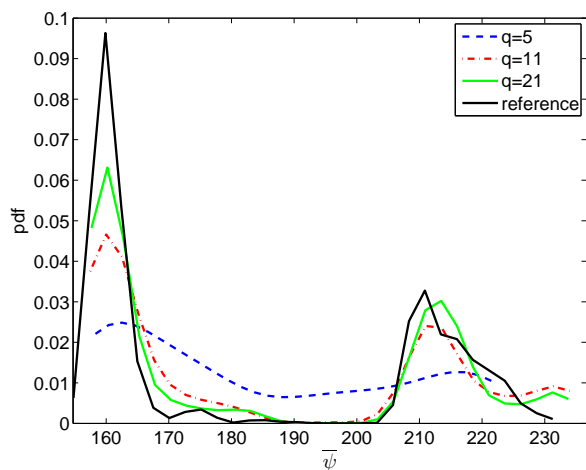

(a) $\operatorname{Pdf}$ of $\bar{\psi}$.

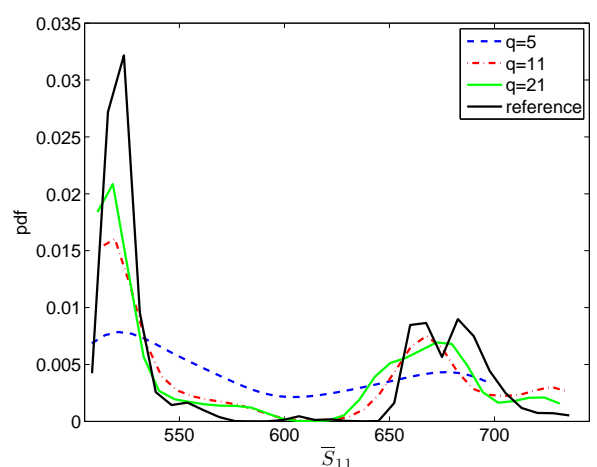

(b) Pdf of $\bar{S}_{11}$

Figure 12. Probability density functions (Pdf) of $\bar{\psi}(\overline{\boldsymbol{C}}, \boldsymbol{\zeta})$ and $\bar{S}_{11}(\overline{\boldsymbol{C}}, \boldsymbol{\zeta})$ corresponding to $\bar{C}_{11}=1.5$, $\bar{C}_{22}=1.5$ and $\bar{C}_{12}=0.5$, for $q=5, q=11$ and $q=21$.

where $J_{q}$ is a quantity of interest corresponding to the post-processing of the S-NEXP solution related to a particular number $q$ and $J_{r e f}$ is the reference quantity of interest. To achieve this computation, we use the set $\left\{\boldsymbol{\zeta}^{(k)}\right\}_{k=1}^{\mu}$ and for each $\boldsymbol{\zeta}^{(k)}$ we associate a realization of the macroscopic strain state following the uniform distribution. Figure 16 illustrates the convergence of the error indicator $\epsilon_{q}$ for different quantities of interest (e.g. $\bar{\psi}, \bar{S}_{11}, \bar{S}_{22}$ and $\bar{S}_{12}$ ) according to $q$. Since the number of discretization points $p$ (related to the macroscopic strains domain $\Delta_{\bar{C}}$ ) is here fi ed, it is not possible to reach a level of error close to zero, which would theoretically be 


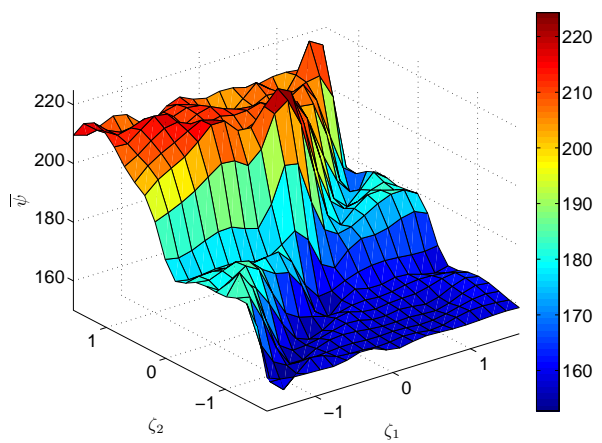

(a) S-NEXP response surface of $\bar{\psi}$ for $q=21$.

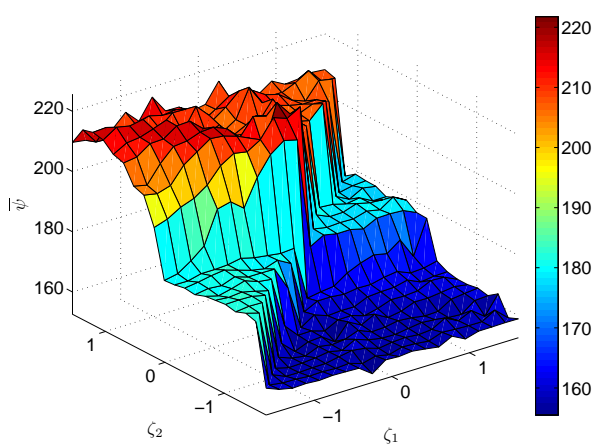

(b) Reference response surface.

Figure 13. Response surfaces of $\bar{\psi}(\overline{\boldsymbol{C}}, \boldsymbol{\zeta})$ according to $\zeta_{1}$ and $\zeta_{2}$ for $\bar{C}_{11}=1.5, \bar{C}_{22}=1.5$ and $\bar{C}_{12}=0.5$.

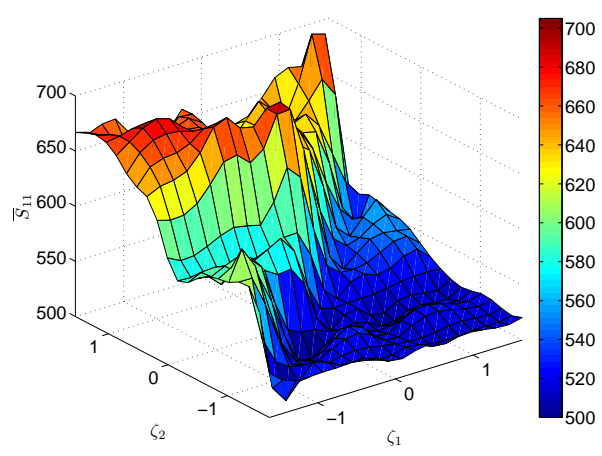

(a) S-NEXP response surface of $\bar{S}_{11}$ for $q=21$.

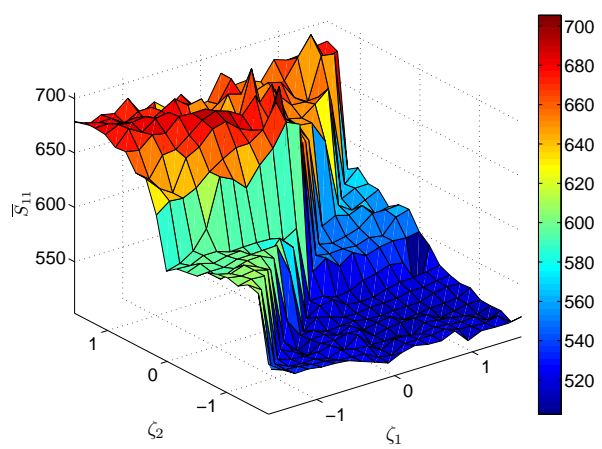

(b) Reference response surface.

Figure 14. Response surfaces of $\bar{S}_{11}(\overline{\boldsymbol{C}}, \boldsymbol{\zeta})$ according to $\zeta_{1}$ and $\zeta_{2}$ for $\bar{C}_{11}=1.5, \bar{C}_{22}=1.5$ and $\bar{C}_{12}=0.5$.

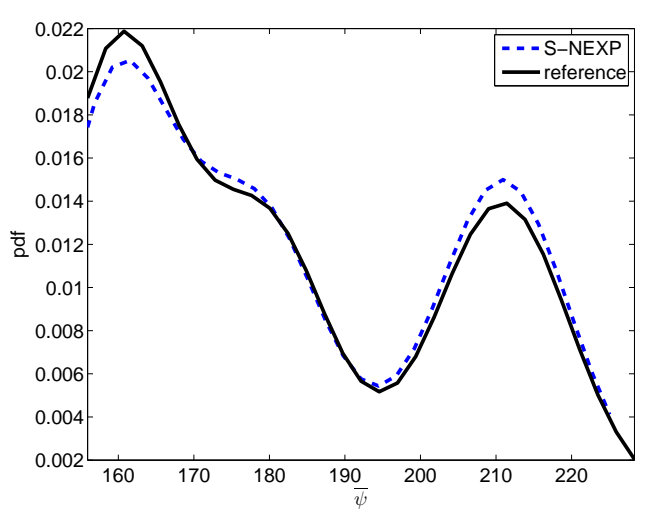

(a) $\operatorname{Pdf}$ of $\bar{\psi}$.

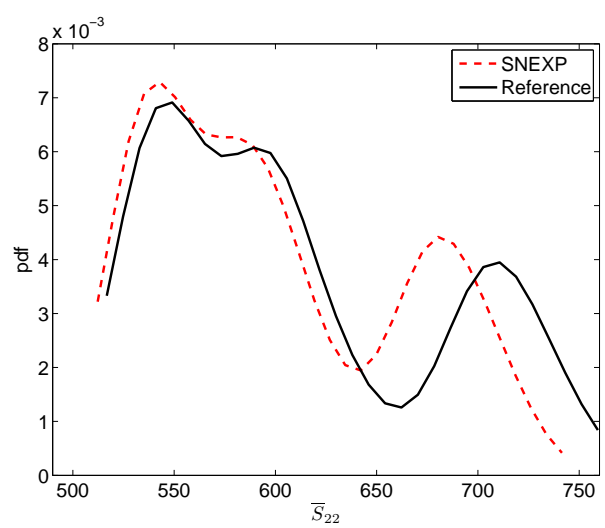

(b) Pdf of $\bar{S}_{22}$.

Figure 15. Probability density functions (Pdf) of $\bar{\psi}$ and $\bar{S}_{22}$ for $\bar{C}_{11}=1.5 \bar{C}_{22}=1.5$ and $\bar{C}_{12}=0.5$.

reached with the increase of $p$. However, we can observe a good and fast convergence of $\epsilon_{q}$. Thus, even for $q=5$, we notice a low level of error slightly superior to $10^{-3}$ for $\bar{\psi}, \bar{S}_{11}$ and $\bar{S}_{22}$, and close to $7.10^{-2}$ for $\bar{S}_{12}$. Figure 17 displays the probability density functions, in semi-log 10 scale, of the sets of realizations used in the computation of the error indicator $\epsilon_{q}$ for $\bar{\psi}$ and $\bar{S}_{11}$. We can notice a very good match of the results for any number of discretization points $q$ except for the probability 


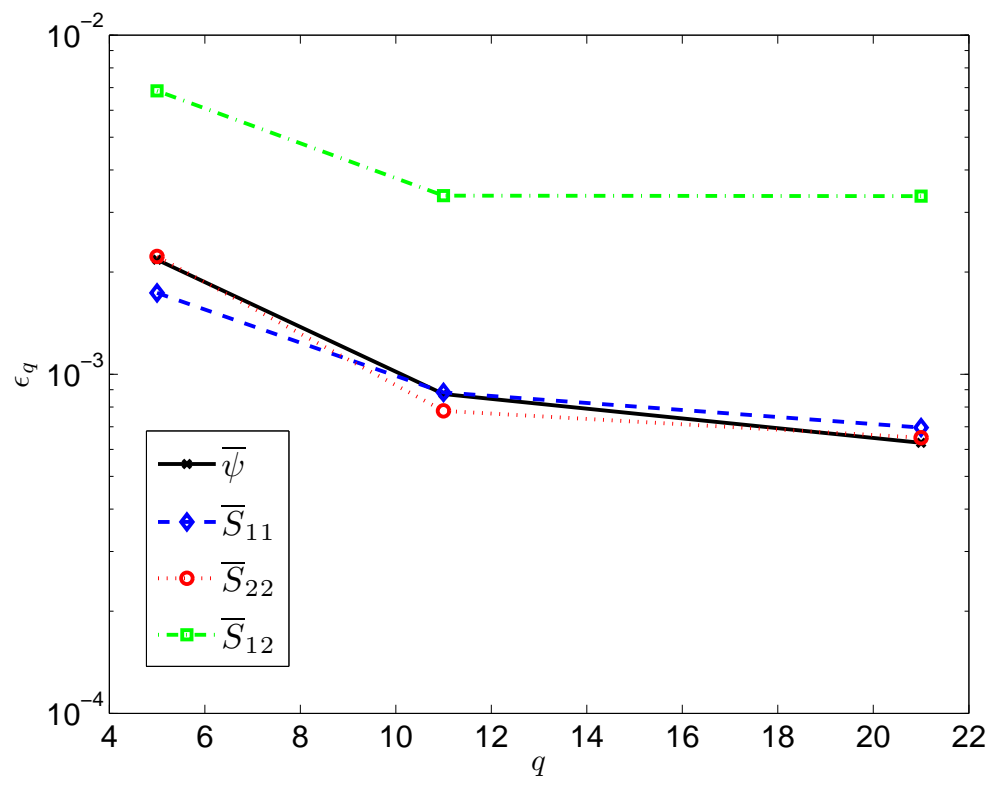

Figure 16. Convergence of error indicator $\epsilon_{q}$ with respect of number of discretization points $q$ with $q=5,11$ and 21 .

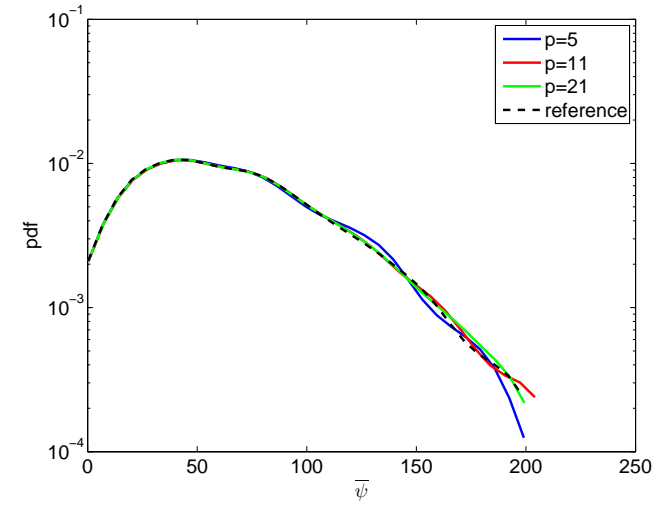

(a) Pdf of $\bar{\psi}$.

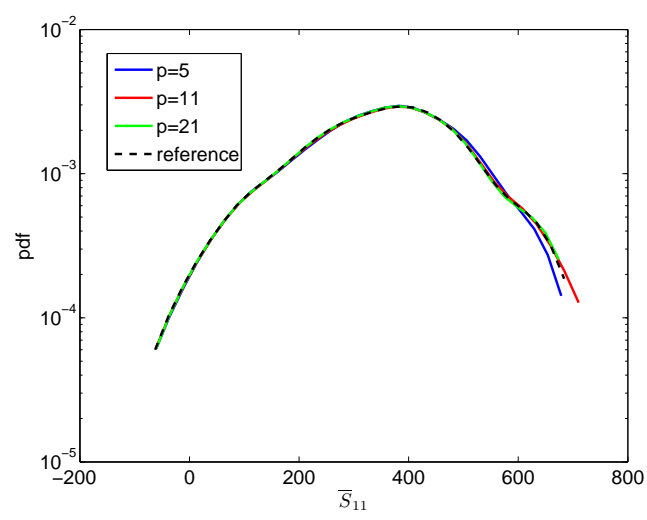

(b) Pdf of $\bar{S}_{11}$.

Figure 17. Comparison of the probability density functions (Pdf) of the S-NEXP solutions and the reference solutions computed for the error analysis.

tails for which only the S-NEXP solution corresponding to $q=21$ matches precisely the reference solution.

We finall focus on the computational costs of the S-NEXP approach. Figure 18 displays the number $Q=p^{D} \times q^{M}$ of nonlinear FEM computations needed in the S-NEXP method with respect to number $M$ of random variables $\zeta_{j}$. On this figure $p=5, D=3$ and the three lines correspond to $q=5, q=11$ and $q=21$. This figur gives an information about the range of applicability of the method proposed. We indeed can observe that the number $Q$ of nonlinear FEM computation becomes very high when $M$ increases. In the case of high dimension (i.e. high values of $M$ ), the involved computational time would become too important to use this kind of method even with a very efficien solver for the nonlinear boundary value problems. However, the proposed approach remains suitable for low or middle dimension. All the computations have been made on a 40-cores computer and the CPU times for the results given in this example are the following ones:

- $M=1: 15$ minutes for $q=5,30$ minutes for $q=11$ and 70 minutes for $q=21$. 


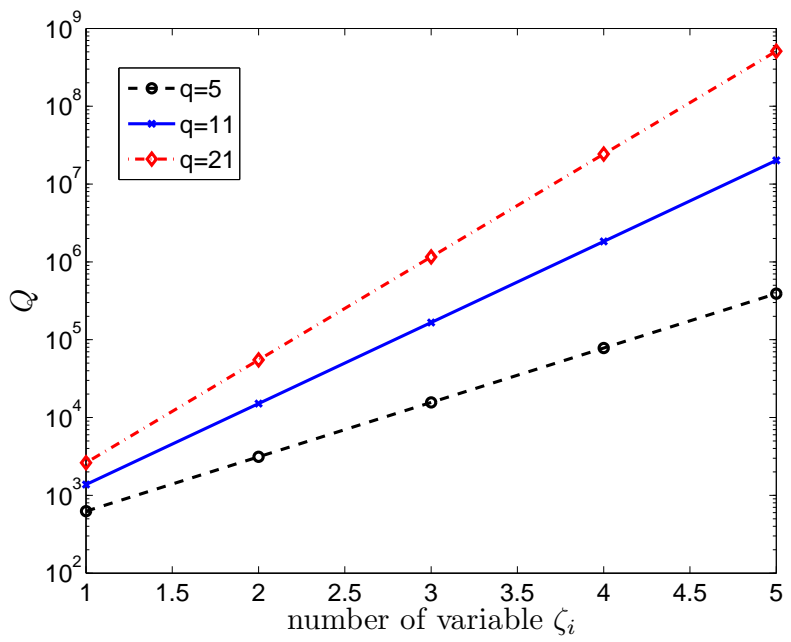

Figure 18. Number $Q$ of nonlinear FEM computations needed in the S-NEXP method as a function of number $M$ of random variables for different number of discretization points $q=(1,11,21)$.

- $M=2: 7$ hours for $q=11$ and 1 day for $q=21$.

\subsection{Numerical example 2: a two scales structural problem}

5.2.1. Problem definitio In this example, we propose to study the efficien y of the S-NEXP method with a two scales structural problem depicted in figur 19(a). We consider a structure made of a heterogeneous hyperelastic material where the geometry of the microstructure is random. The microscopic problem corresponds to the one studied in Section 5.1 with the parameters of the probabilistic content given in Section 5.1.1. To characterize the strain energy density function representing the macroscopic constitutive behavior of the material, we use the S-NEXP solutions with the different discretizations (e.g. $q=5, q=11$ and $q=21$ ). At the macroscopic level, the dimensions of the structure are $L=1 \mathrm{~m}, H=1 \mathrm{~m}$ and $e=0.2 \mathrm{~m}$ and displacements $\Delta \overline{\boldsymbol{u}}=5 \%$ are imposed on a part $\bar{\Gamma}_{\bar{u}}$ of the structural domain. For the macroscopic space approximation, we use a mesh composed of 372 3-nodes triangular finit elements depicted on figur 19(b). To test the efficien y of the method, we build reference solutions with a multilevel Finite Element Method, denoted $\mathrm{FE}^{2}$ in the following (see $[7,9]$ ), with the same macroscopic mesh used for the S-NEXP calculations.

5.2.2. Accuracy of the S-NEXP solution We propose to show the efficien y of the S-NEXP method with post-processing of the macroscopic solution. During the macroscopic computation, each integration point in the macroscale discretization must be associated with a microstructure. Two cases are considered.

1. Case (i): the same geometry of the microstructure corresponding to the statistical mean of the stochastic model of the geometry of the microstructure, is used for each integration point.

2. Case (ii): the random geometry of the microstructure constructed with the realizations $\left\{\boldsymbol{\zeta}^{(k)}\right\}_{k=1}^{\mu}$ is used for each integration point.

Case (ii) takes into account the stochastic fluctuation which are significan as shown in Section 5.1. It is worth noticing that the post-processing of the S-NEXP solution is very fast thanks to the explicit continuous form of the potential (less than one minute with a laptop computer for this example) while a complete $\mathrm{FE}^{2}$ computation takes more than one hour with a computer cluster with 40 cores. Figure 20 displays the von Mises stresses for the macroscopic computations corresponding to the three S-NEXP solution (numerical potential computed with $q=5, q=11$ and $q=21$ ) and the $\mathrm{FE}^{2}$ reference solution for case (i). We can observe a good agreement of the results even for a small 


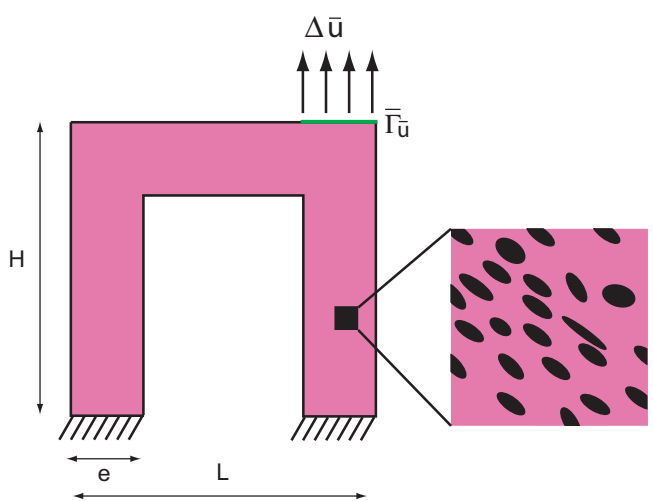

(a)

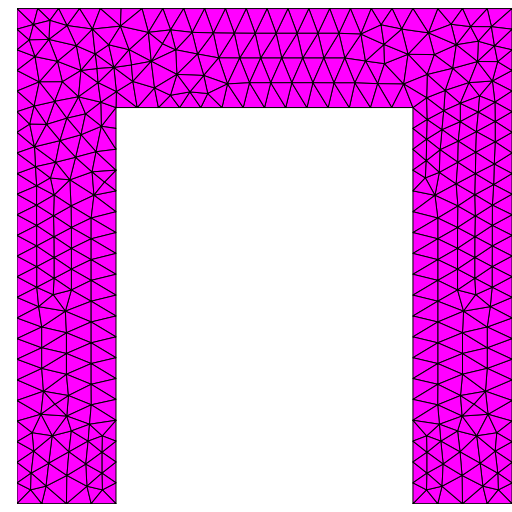

(b)

Figure 19. Geometry and boundary conditions for the two scales structural problem (a); Finite element mesh associated with the microstructure (b).

number $q$ of discretization points. In the same manner, figur 21 displays the von Mises stresses for case (ii). We can also notice a good convergence of the S-NEXP solution towards the reference solution even if the macroscopic solution is not smooth according to the von Mises stress field However, we can observe that the convergence is slower than the one corresponding to case $(i)$ : this is due to the important variations of the potential which are bad approximated with a low number $q$ of discretization points has shown on figur 9. Thus, a good macroscopic solution required to use a number $q$ of discretization points which is high enough to capture these irregularities. Finally, figur 22 shows the typical convergence of the macroscopic Newton-Raphson algorithm for both cases. We can observe that behavior of the macroscopic computations are the same for the two cases and thus that the second-order convergence is still reached even for case (ii).

\section{CONCLUSIONS}

To the knowledge of the authors, the present paper is the firs one devoted to the challenging issues concerning the computational nonlinear stochastic homogenization using a non-concurrent multiscale approach for hyperelastic heterogeneous microstructures analysis. The non-concurrent multiscale approach for micro-macro nonlinear mechanics has been validated for the deterministic case in the literature. In this work, we have extended and validated this approach to the stochastic case for hyperelastic heterogeneous microstructures. Since the non-concurrent multiscale approach is based on the use of a tensorial decomposition, which is then submitted to the curse of dimensionality, we have performed an analysis with respect to the stochastic dimension. In order to show the capability of this multiscale approach in the stochastic framework, a prior stochastic model of the microstructure has been introduced, which allows the efficien y to be analyzed with respect to the stochastic dimension. Clearly, such a prior stochastic model could be replaced by any other model which would have the same capabilities. In this work, we have considered a fibe reinforced polymer composites for which the geometry of the microstructure is random. The firs point of the proposed method has consisted in building a stochastic model of the random geometry for the microstructure. A major difficult is related to the impenetrability condition between the fiber and the stochastic dimension induced by the number of fibers The second point is devoted to the extension of the method of numerical explicit potentials to the stochastic framework. The macroscopic constitutive behavior of the material is define by a continuous explicit form of the macroscopic strain energy density function (potential) according to the macroscopic strain state and the random geometrical parameters. A separated variables representation associated with an interpolation scheme has allowed obtaining the continuous explicit form of the effective 


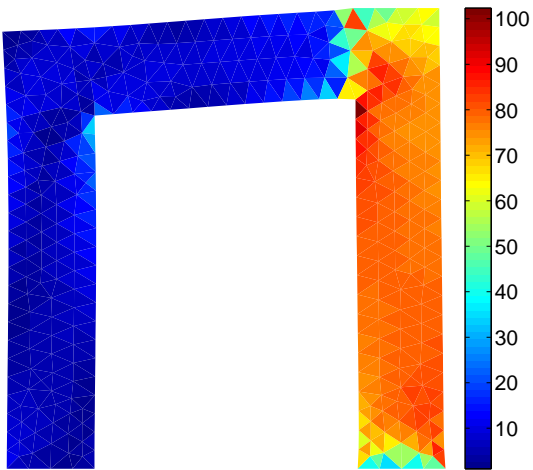

(a) VM stresses for $q=5$.

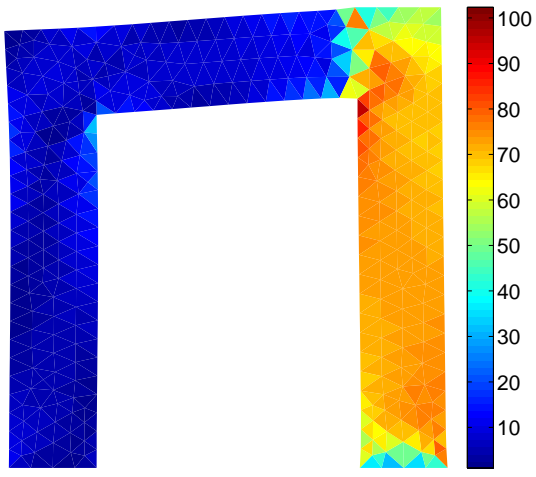

(c) VM stresses for $q=21$.

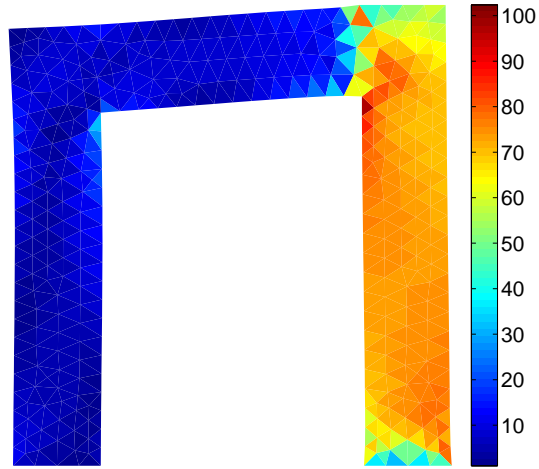

(b) VM stresses for $q=11$.

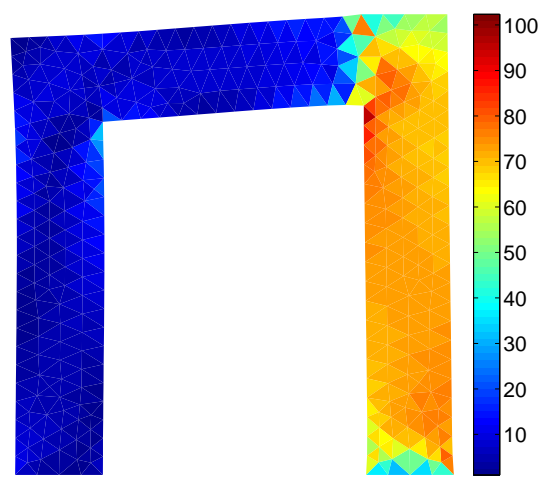

(d) VM stresses for the $\mathrm{FE}^{2}$ reference solution.

Figure 20. Comparison of the von Mises stress field for case (i); mean geometry of the microstructure applied to each integration point.

potential which, by derivation, has allowed determining the components of the stress and the tangent elastic tensors required for the macroscopic computations. Two numerical examples have been presented to validate the proposed approach. However, in the development of the proposed approach, several difficultie have arisen and must be pointed out. First, as the strain energy density function holds irregularities with respect of the geometrical parameters, an important number of discretization points must be employed to capture these local gradients. Thus, the use of a uniform discretization for the parameters domain does not appear as the best choice since unnecessary points are introduced. A non-uniform discretization strategy based, for instance, on the volume fraction will be investigated in a forthcoming paper. Moreover, as the main computational cost comes from the construction of the database, an optimal discretization strategy would allow reducing these costs. Finally, we pointed out that those high computational times prevent from using the approach with an important number of reduced random variables characterizing the geometry of the microstructure. This limitation is classical in the context of parametric stochastic methods when one increases the stochastic dimension. Further works dedicated to circumvent this drawback will be investigated in forthcoming papers.

\section{ACKNOWLEDGEMENTS}

This research was funded by the French Research Agency (Agence Nationale de la Recherche) under TYCHE contract ANR-2010-BLAN-0904. 


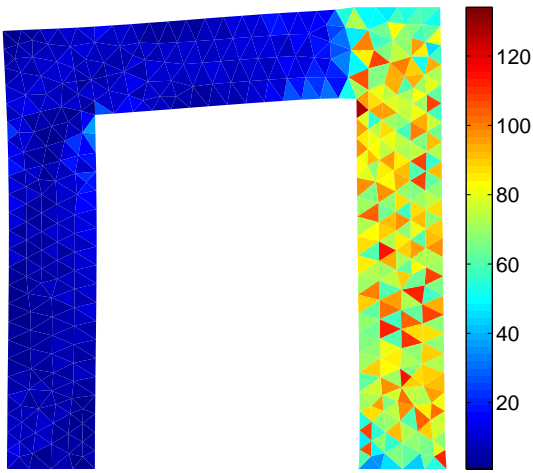

(a) VM stresses for $q=5$.

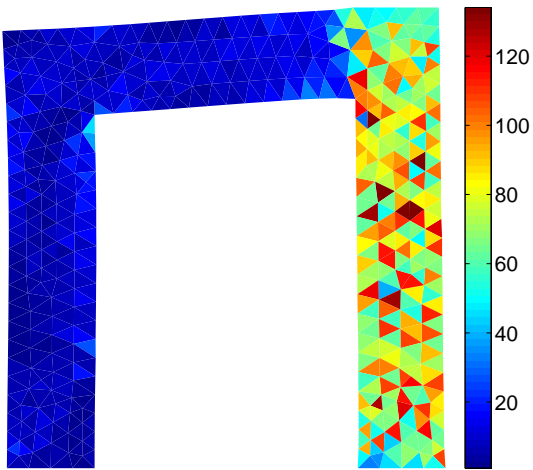

(c) VM stresses for $q=21$.

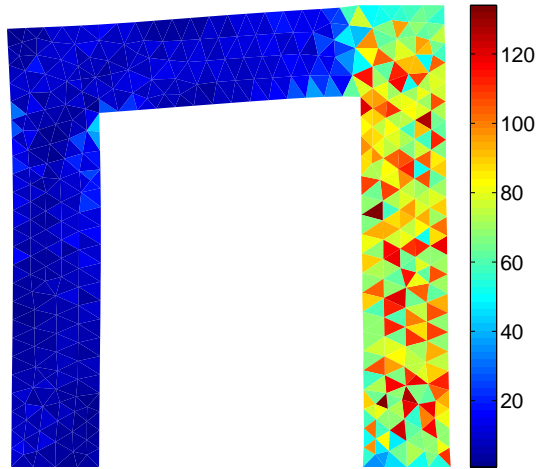

(b) VM stresses for $q=11$

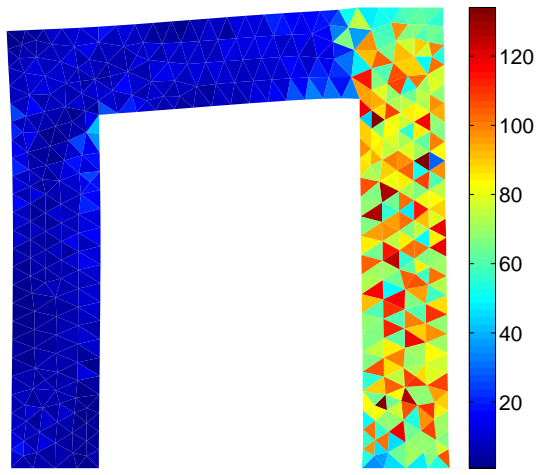

(d) VM stresses for the $\mathrm{FE}^{2}$ reference solution.

Figure 21. Comparison of the von Mises stress field for case (ii): particular realization of the geometry of the microstructure applied to each integration point.

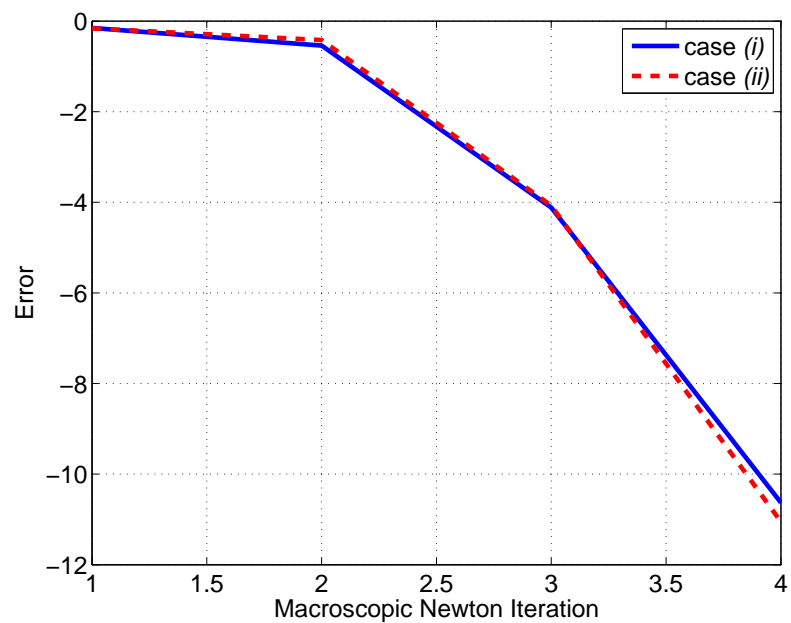

Figure 22. Convergence of the macroscopic Newton-Raphson algorithm for case (i) and case (ii).

\section{REFERENCES}

1. deBotton G. Transversally isotropic sequentially laminated composites in finit elasticity. J. Mech. Phys. Solids 2005; 53(6):1334-1361. 
2. Aboudi J. Finite strain micromechanical modeling of multiphase composites. Int. J. Multiscale Comput. Eng. 2008; 6(5):411-434.

3. Ghosh S., Lee K., Raghavan P. A multilevel computational model for multi-scale damage analysis in composite and porous media. Int. J. Solids Struct. 2001; 38:2335-2385.

4. Ponte-Castañeda P., Tiberio E. Second-order homogenization method in finit elasticity and applications to blackfille elastomers. J. Mech. Phys. Solids 2000; 48(6-7):1389-1411.

5. Lopez-Pamies O., Ponte Castañeda P. Second-Order Estimates for the Macroscopic Response and Loss of Ellipticity in Porous Rubbers at Large Deformations. J. Elast. 2004; 76(3):247-287.

6. Smit R., Brekelmans W., Meijer H. Prediction of the mechanical behavior of nonlinear heterogeneous systems by multi-level finit element modeling. Comput. Methods Appl. Mech. Engng. 1998; 155:181-192.

7. Feyel F., Chaboche J.L. FE2 multiscale approach for modelling the elastoviscoplastic behaviour of long fibe $\mathrm{SiC} / \mathrm{Ti}$ composite materials. Comput. Meth. Appl. Mech. Eng. 2000; 183:309-330.

8. Terada K., Kikuchi N. A class of general algorithms for multi-scale analysis of heterogeneous media. Comput. Methods Appl. Mech. Engng. 2001; 190:5427-5464.

9. Feyel F. A multilevel finit element method $\left(\mathrm{FE}^{2}\right)$ to describe the response of highly non-linear structures using generalized continua. Comput. Methods Appl. Mech. Engng. 2003; 192:3233-3244.

10. Kouznetsova V.G., Geers M.G.D, Brekelmans W.A.M. Multi-scale second order computational homogenization of multi-phase materials: a nested finit element solution strategy. Comput. Methods Appl. Mech. Engng. 2004; 193:5525-5550.

11. Yvonnet J., He Q.C. The Reduced Model Multiscale Method (R3M) for the nonlinear homogenization of hyperelastic media at finit strains. J. of Comput. Physics 2007; 223:341-368.

12. Monteiro E., Yvonnet J., He Q.C. Computational Homogenization for nonlinear conduction in heterogeneous materials using model reduction. Comput. Mater. Sci. 2008; 42:704-712.

13. Nezamabadi S., Yvonnet J, Zahrouni H., Pottier-Ferry M. A multilevel computational strategy for handling microscopic and macroscopic instabilities. Comput. Methods Appl. Mech. Engng. 2009; 198:2099-2110.

14. Ogden R.W., Saccomandi G., Sgura I. Fitting hyperelastic models to experimental data. Comput. Mech. 2004; 34:484-502.

15. Takano N., Zako M., Ohnishi Y. Macro-micro uncoupled homogenization procedure for microscopic nonlinear behavior analysis of composites. Mater. Sci. Res. Int., Comput. Methods Appl. Mech. Eng. 1996; 2(2):81-86.

16. Temizer I.,Wriggers P. An adaptive method for homogenization in orthotropic nonlinear elasticity. Comput. Methods Appl. Mech. Eng. 2007; 35-36:3409-3423.

17. Yvonnet J., Gonzalez D., He Q.C. Numerically explicit potentials for the homogenization of nonlinear elastic heterogeneous materials. Comput. Methods Appl. Mech. Engng. 2009; 198:2723-2737.

18. Yvonnet J., He Q.C. A non-concurrent multiscale method for computing the response of nonlinear elastic heterogeneous structures. European Journal of Computational Mechanics 2010; 19:105-116.

19. Yvonnet J., Monteiro E., He Q.C. Computational homogenization of hyperelastic heterogeneous structures: a nonconcurrent approach. Submitted for publication.

20. Torquato S. Random Heterogeneous Materials: Microstructures and Macroscopic Properties. Springer: New-York, 2002.

21. Koutsourelakis P.S., Deodatis G. Simulation of Binary Random Fields with Applications to Two-Phase Random Media. Journal of Engineering Mechanics 2005, 131(4):397-412.

22. Soize C. A nonparametric model of random uncertainties on reduced matrix model in structural dynamics. Probabilist. Engrg. Mech. 2000; 15(3):277-294.

23. Soize C. Maximum entropy approach for modeling random uncertainties in transient elastodynamics. J. Acoust. Soc. Am. 2001; 109(5):1979-1996.

24. Soize C. Tensor-valued random field for meso-scale stochastic model of anisotropic elastic microstructure and probabilistic analysis of representative volume element size. Probabilist. Engrg. Mech. 2008; 23:307-323.

25. Guilleminot J., Noshadravan A., Soize C., Ghanem R.G. A probabilistic model for bounded elasticity tensor random field with application to polycrystalline microstructures. Comput. Methods Appl. Mech. Eng. 2011; 200:1637-1648.

26. Jardak M. Ghanem R.G. Spectral stochastic homogenization of divergence-types pdes. Comput. Methods Appl. Mech. Engng. 2004; 193(6-8):429-447.

27. Frank Xu X. A multiscale stochastic finit elment method on elliptic problems involving uncertainties. Comput. Methods Appl. Mech. Engng. 2007; 196:2723-2736.

28. Tootkaboni M., Graham-Brady L. A multi-scale spectral stochastic method for homogenization of multi-phase periodic composites with random material properties Int. J. Numer. Meth. Engng. 2010; 83:59-90.

29. Spanos P.D., Kontsos A. A multiscale Monte Carlo finit element method for determining mechanical properties of polymer nanocomposites. Probabilistic Engineering Mechanics 2008, 23:456-470.

30. Hiriyur B., Waisman H., Deodatis G. Uncertainty quantificatio in homogenization of heterogeneous microstructures modeled by XFEM. Int. J. Numer. Meth. Engng. 2011; doi: 10.1002/nme.3174.

31. Jeulin D. Random texture models for material structures. Statistics and Computing 2000: 10:121-132.

32. Jeulin D. Modelling random media. Image Analysis and Stereology 2002; 21:S31-S40.

33. Grigoriu M. Level-cut inhomogeneous filtere Poisson fiel for the two-phase microstructures. Int. J. Numer. Meth. Engng. 2009; 78:215-228.

34. Rahman S. A random fiel model for generating synthetic microstructures of functionally graded materials. Int. J. Numer. Meth. Engng. 2008; 76:972-993.

35. Loève M. Probability Theory. I, fourth edition, in: Graduate Texts in Mathematics, vol. 45. Springer-Verlag, NewYork, 1977.

36. Tran A.B., Yvonnet J., Toulemonde C., Sanahuia J. A multiple level set approach to prevent numerical artefacts in complex microstructures with nearby inclusions within XFEM.Int. J. Numer. Meth. Engng. 2011; 85(11):1436-1459.

37. Kiers H.A.L. Toward a standardized notation and terminology in multiway analysis. J. Chemometr. 2000; 14:105122. 
38. Carol J.D., Chang J.J. Analysis of individual differences in multidimensional scaling via an N-way generalization of 'Eckart-Young' decomposition. Psychometrika, 1970; 35:283-319.

39. Zhang T., Golub G.H. Rank-one approximation to high order tensor. SIAM J. Matrix Anal. Appl. 2001; 23(2):534550

40. MATLAB Tensor Toolbox Version 2.4 (released Mar. 22, 2002) Sandia National Laboratories, http://csmr.ca.sandia.gov/tgkolda/TensorToolbox/

41. Beatson R.K. On the convergence of some cubic spline interpolation schemes. SIAM J. Numer. Anal. 1986; 23(4):903-912 